\title{
A Giant Sample Of Giant Pulses From The Crab Pulsar
}

\author{
M. B. Mickaliger \\ M.A. McLaughlin \\ D. R. Lorimer \\ G. I. Langston
}

A. V. Bilous

See next page for additional authors

Follow this and additional works at: https://researchrepository.wvu.edu/faculty_publications

\section{Digital Commons Citation}

Mickaliger, M. B.; McLaughlin, M. A.; Lorimer, D. R.; Langston, G. I.; Bilous, A. V.; Kondratiev, V. I.; Lyutikov, M.; Ransom, S. M.; and Palliyaguru, N., "A Giant Sample Of Giant Pulses From The Crab Pulsar" (2012). Faculty Scholarship. 443.

https://researchrepository.wvu.edu/faculty_publications/443 
Authors

M. B. Mickaliger, M. A. McLaughlin, D. R. Lorimer, G. I. Langston, A. V. Bilous, V. I. Kondratiev, M. Lyutikov, S. M. Ransom, and N. Palliyaguru 


\title{
A GIANT SAMPLE OF GIANT PULSES FROM THE CRAB PULSAR
}

\author{
M. B. Mickaliger ${ }^{1}$, M. A. McLaughlin ${ }^{1,8}$, D. R. Lorimer $^{1,8}$, G. I. Langston ${ }^{2}$, A. V. Bilous ${ }^{3}$,

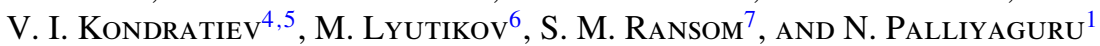 \\ ${ }^{1}$ Department of Physics, West Virginia University, Morgantown, WV 26506, USA \\ ${ }^{2}$ National Radio Astronomy Observatory, Green Bank, WV 24944, USA \\ ${ }^{3}$ Department of Astronomy, University of Virginia, P.O. Box 400325, Charlottesville, VA 22904, USA \\ ${ }^{4}$ Netherlands Institute for Radio Astronomy (ASTRON), Postbus 2, 7990 AA Dwingeloo, The Netherlands \\ ${ }^{5}$ Astro Space Center of the Lebedev Physical Institute, Profsoyuznaya str. 84/32, Moscow 117997, Russia \\ ${ }^{6}$ Department of Physics, Purdue University, 525 Northwestern Avenue, West Lafayette, IN 47907-2036, USA \\ ${ }^{7}$ National Radio Astronomy Observatory, Charlottesville, VA 22903, USA \\ Received 2012 April 5; accepted 2012 October 1; published 2012 November 5
}

\begin{abstract}
We observed the Crab pulsar with the $43 \mathrm{~m}$ telescope in Green Bank, WV over a timespan of 15 months. In total we obtained $100 \mathrm{hr}$ of data at $1.2 \mathrm{GHz}$ and seven hours at $330 \mathrm{MHz}$, resulting in a sample of about 95,000 giant pulses (GPs). This is the largest sample, to date, of GPs from the Crab pulsar taken with the same telescope and backend and analyzed as one data set. We calculated power-law fits to amplitude distributions for main pulse (MP) and interpulse (IP) GPs, resulting in indices in the range of 2.1-3.1 for MP GPs at $1.2 \mathrm{GHz}$ and in the range of 2.5-3.0 and 2.4-3.1 for MP and IP GPs at $330 \mathrm{MHz}$. We also correlated the GPs at $1.2 \mathrm{GHz}$ with GPs from the Robert C. Byrd Green Bank Telescope (GBT), which were obtained simultaneously at a higher frequency $(8.9 \mathrm{GHz})$ over a span of $26 \mathrm{hr}$. In total, $7933 \mathrm{GPs}$ from the $43 \mathrm{~m}$ telescope at $1.2 \mathrm{GHz}$ and 39,900 GPs from the GBT were recorded during these contemporaneous observations. At $1.2 \mathrm{GHz}, 236$ (3\%) MP GPs and 23 (5\%) IP GPs were detected at $8.9 \mathrm{GHz}$, both with zero chance probability. Another $15(4 \%)$ low-frequency IP GPs were detected within one spin period of high-frequency IP GPs, with a chance probability of $9 \%$. This indicates that the emission processes at high and low radio frequencies are related, despite significant pulse profile shape differences. The $43 \mathrm{~m}$ GPs were also correlated with Fermi $\gamma$-ray photons to see if increased pair production in the magnetosphere is the mechanism responsible for GP emission. A total of 92,022 GPs and $393 \gamma$-ray photons were used in this correlation analysis. No significant correlations were found between GPs and $\gamma$-ray photons. This indicates that increased pair production in the magnetosphere is likely not the dominant cause of GPs. Possible methods of GP production may be increased coherence of synchrotron emission or changes in beaming direction.
\end{abstract}

Key words: gamma rays: general - pulsars: individual (PSR B0531+21) - radiation mechanisms: non-thermal

\section{INTRODUCTION}

The Crab pulsar was discovered by Staelin \& Reifenstein in (1968) through its giant pulses. Giant pulses (GPs) can be thousands of times brighter than the average pulse. The temporal occurrence of GPs is random but at frequencies below $3 \mathrm{GHz}$ they always occur at the phase of either the Crab pulsar's main pulse (MP) or interpulse (IP; Lundgren 1994). At frequencies from $4 \mathrm{GHz}$ to $8.4 \mathrm{GHz}$, GPs are emitted at the phases of the MP and IP as well as at the phases of two additional high-frequency components (Moffett \& Hankins 1996). Above $8.5 \mathrm{GHz}$, GPs are again seen at only the phases of the MP and IP (Jessner et al. 2010)

The emission mechanism of GPs is still an open question (e.g., Petrova 2006; Istomin 2004; Weatherall 1998). GPs could be caused by increased pair production in the magnetosphere, increased coherence of synchrotron emission, or changes in beaming direction. Correlating radio GPs with high-energy photons is one way to determine if increased pair production is a major cause of GPs. A recent model (Lyutikov 2007) proposes that GPs are generated on the last closed magnetic field line near the light cylinder via anomalous cyclotron resonance. If this is true, there would be an increase in $\gamma$-rays at the times of radio GPs. Anomalous cyclotron resonances have been previously proposed as a cause of GP emission by Lyutikov et al. (1999)

\footnotetext{
8 Also adjunct at the National Radio Astronomy Observatory, Green Bank, WV 24944, USA.
}

and Machabeli \& Usov (1979). We expect the $\gamma$-rays to be phase aligned with the radio GPs at $1.2 \mathrm{GHz}$ as the $\gamma$-ray and $1.2 \mathrm{GHz}$ radio profiles are aligned (see Figure 1). Since we have such a large data set of GPs, we can correlate them with $\gamma$-ray photons to test this model. Correlations between radio GPs and $\gamma$-ray photons have been carried out previously by Bilous et al. (2011) and Lundgren et al. (1995). Shearer et al. (2003) correlated radio GPs and optical photons and found a slight correlation, and work by Collins et al. (2012) supports this result.

The Fermi Large Area Telescope (LAT) is a pair conversion telescope that operates in the energy range from $20 \mathrm{MeV}$ to $300 \mathrm{GeV}$. It has a large field of view (2.4 sr), which allows it to rapidly map the entire sky, and very good angular resolution, minimizing background contamination (Atwood et al. 2009). The Crab pulsar is a bright Fermi source and has been studied at these energies. Abdo et al. (2010) found that the $\gamma$-ray profile is double peaked and matches the $1.4 \mathrm{GHz}$ profile, with the $\gamma$-ray peaks leading the radio peaks by $\sim 0.01$ phase. Since the LAT covers the energies predicted by Lyutikov (2007) and observes the Crab pulsar multiple times per day, we can use Fermi data to test Lyutikov's theory.

The power-law nature of the amplitude distribution of GPs is well known (e.g., Argyle \& Gower 1972; Popov \& Stappers 2007), but there have been varying values for the powerlaw index calculated, even for similar frequencies (e.g., Karuppusamy et al. 2010; Bhat et al. 2008). With our large sample of GPs, we can calculate power-law indices over a long timespan. Since the amplitude distribution of pulses from many 


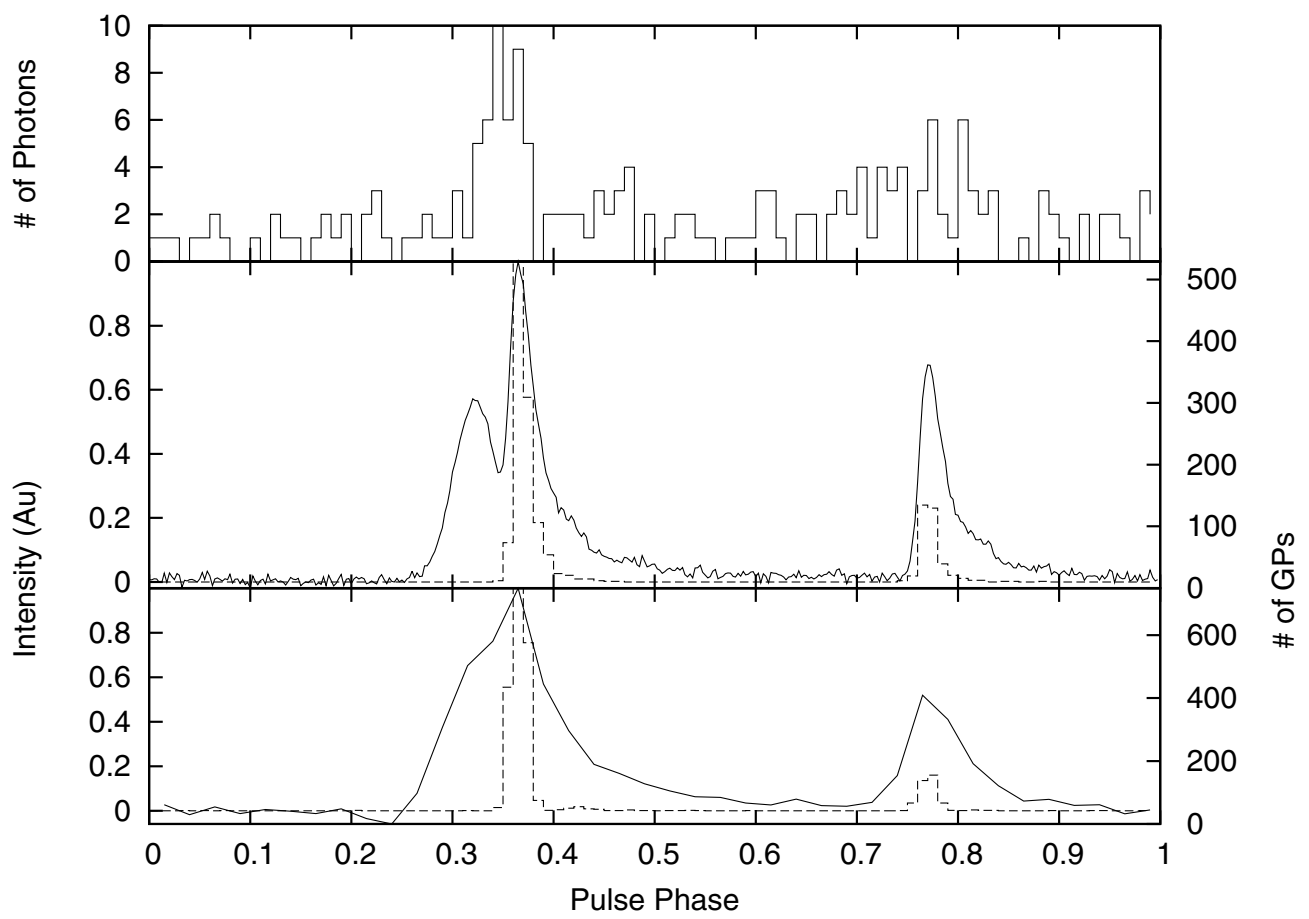

Figure 1. Folded pulse profiles from MJD 55406 for Fermi (top), the GBT (middle, solid line), and the GB43 (bottom, solid line), as well as histograms of the number of GPs for the GBT (middle, dashed line) and GB43 (bottom, dashed line). The Fermi profile is made using photons above $100 \mathrm{MeV}$ over a $24 \mathrm{hr}$ period ( $\sim 11 \mathrm{hr}$ on source time). The GB43 observations were taken at a center frequency of $330 \mathrm{MHz}$ over a $220 \mathrm{MHz}$ band for $3.7 \mathrm{hr}$ and the GBT observations were taken at a center frequency of $350 \mathrm{MHz}$ over a $100 \mathrm{MHz}$ band for 10 minutes. The folded radio profiles have been normalized to have peaks of unity.

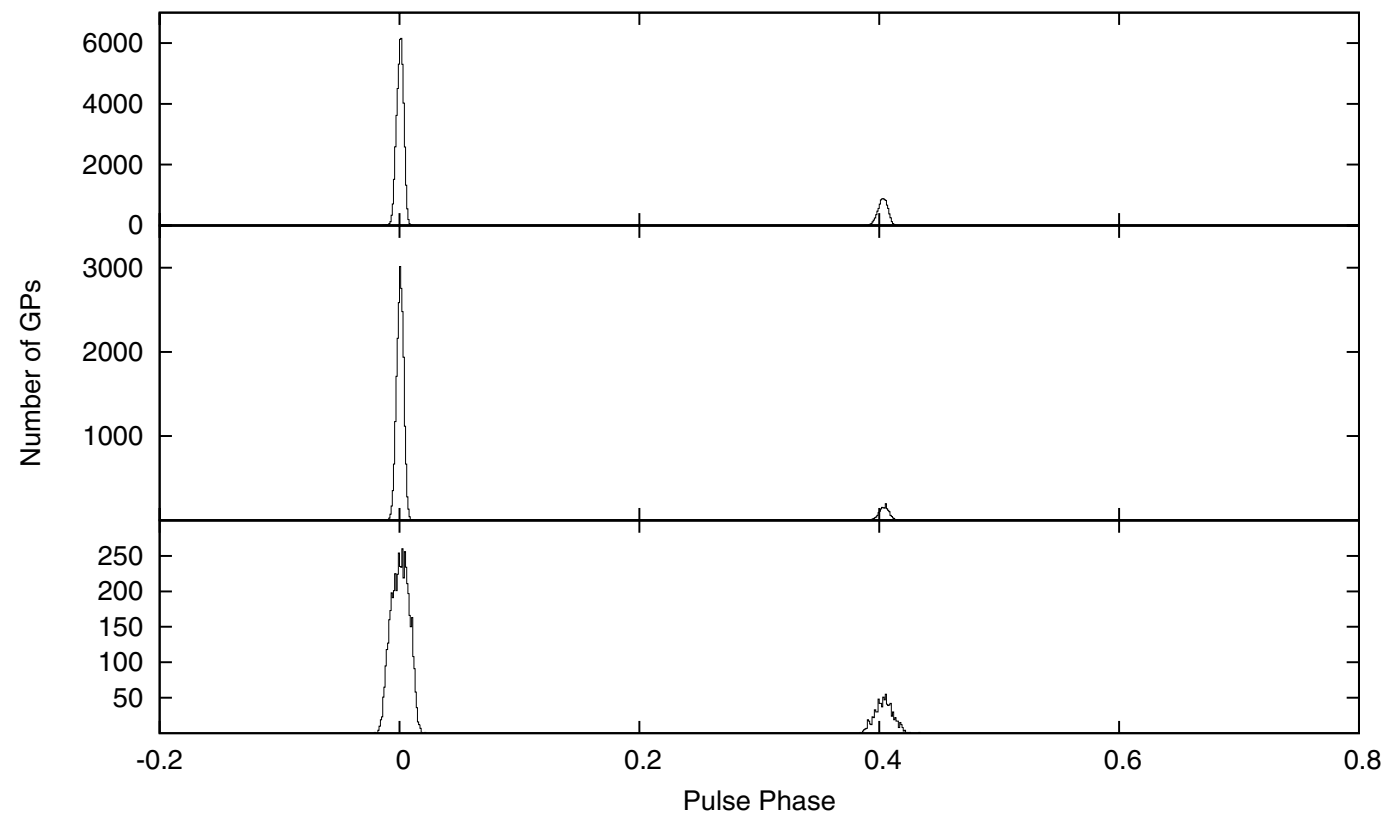

Figure 2. Number of GPs vs. pulse phase for all GPs collected with the GB43 at $1.2 \mathrm{GHz}$ (top, post-flare; middle, pre-flare) and $330 \mathrm{MHz}$ (bottom). Most GPs at frequencies below $5 \mathrm{GHz}$ come at the phase $(\sim 0)$ of the MP, while there are still a considerable number at the IP phase $(\sim 0.4)$. No GPs are seen at other phases. The FWHMs of the $1.2 \mathrm{GHz}$ distributions ( $226 \mu$ s pre-flare, $292 \mu$ s post-flare) are on the order of the FWHMs of the folded profiles at $1.2 \mathrm{GHz}$, while the FWHM of the $330 \mathrm{MHz}$ distribution $(704 \mu \mathrm{s})$ is much narrower than the folded profile and is on the order of the average GP profile at $330 \mathrm{MHz}$. Even though there were only four observing epochs after the flare, there were many more GPs recorded than pre-flare due to a large increase in receiver sensitivity.

pulsars is log-normal (Ritchings 1976), GPs must have a different emission mechanism. By comparing our power-law index with those of other neutron star source classes (pulsars, magnetars, RRATs), we may be able to put constraints on the GP emission mechanism.

A $\gamma$-ray flare was recently observed from the Crab Nebula by the AGILE satellite (Tavani et al. 2011). The flare lasted from
MJDs 55457-55461 and was also observed by Fermi (Hays et al. 2010). This occurred during the span of our observations, so we can compare radio and $\gamma$-ray properties of the pulsar from before the flare with a few days of data taken about two months after the flare to verify that the flare was not associated with the pulsar. Current theories suggest that the flare was caused by electrons accelerated by magnetic reconnection at 


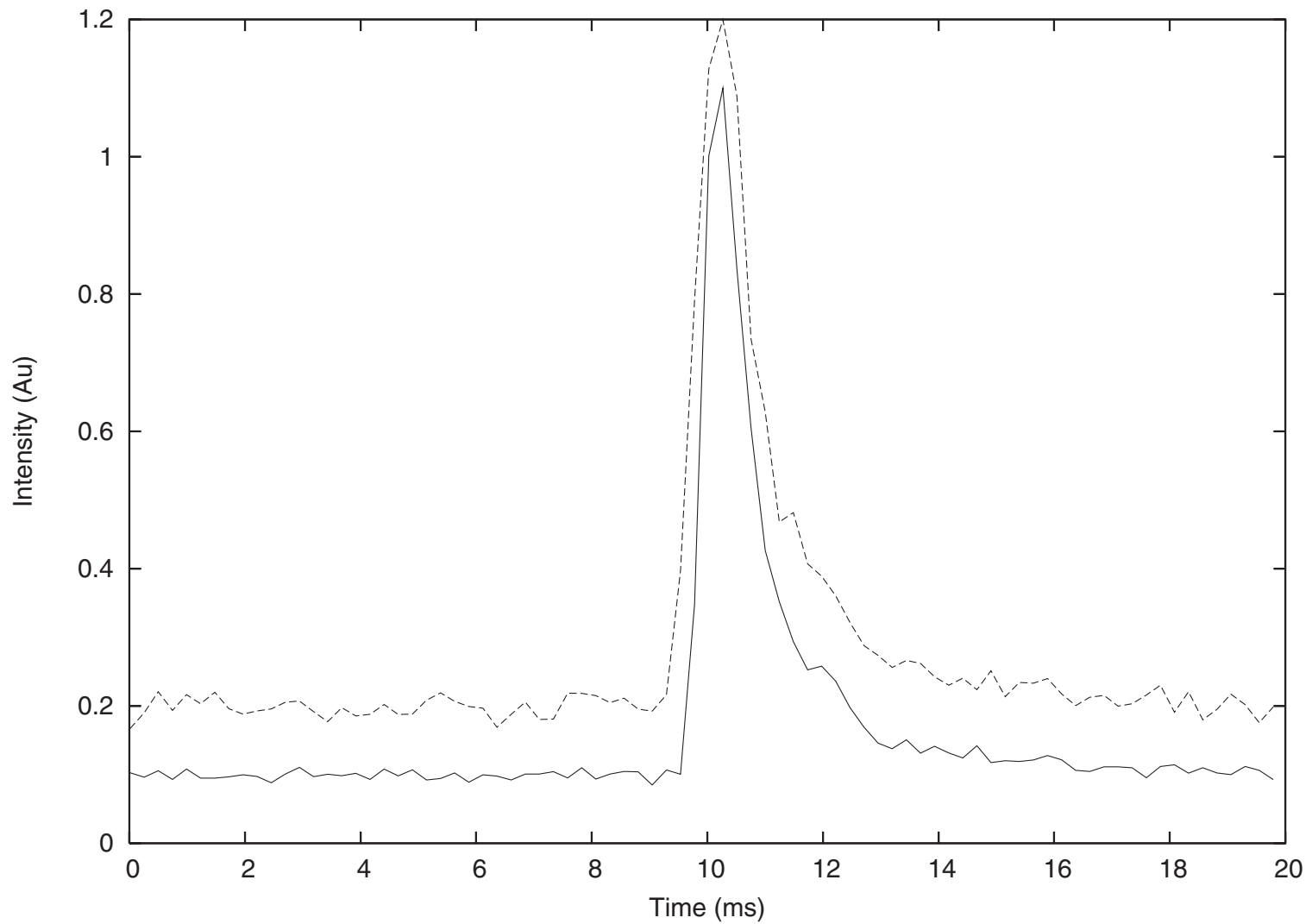

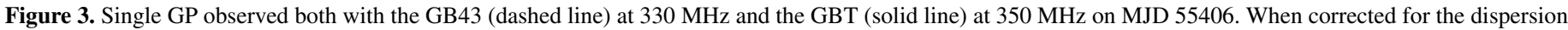

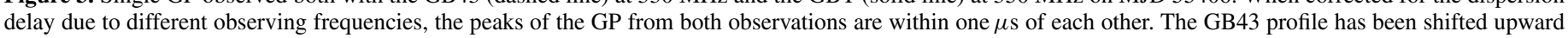
for clarity.

the termination shock of the Crab Nebula (e.g., Bednarek \& Idec 2011; Bykov et al. 2012; Cerutti et al. 2012).

The plan for the rest of this paper is as follows. Section 2 describes the radio observations and outlines the radio analysis. Section 3 discusses the GP statistics and the power-law index calculations. Section 4 details the correlation between $43 \mathrm{~m}$ and Green Bank Telescope (GBT) GPs. Section 5 presents the Fermi data used and its reduction as well as the $43 \mathrm{~m} /$ Fermi correlation. Section 6 discusses the recent $\gamma$-ray flare. Finally, conclusions are offered in Section 7.

\section{RADIO OBSERVATIONS}

We collected data over the course of 15 months using the $43 \mathrm{~m}$ telescope (hereafter GB43), located at the National Radio Astronomy Observatory site in Green Bank, WV. The GB43 is funded by MIT Lincoln Labs to perform bistatic radar observations of the ionosphere (Langston 2007). Most of the observations had a center frequency of $1.2 \mathrm{GHz}$ with a useable bandwidth of $400 \mathrm{MHz}$, with a handful of the later observations centered at $330 \mathrm{MHz}$, with a useable bandwidth of $150 \mathrm{MHz}$. All of the observations were taken using 4096 frequency channels and varying sampling times, listed in Table 1. Some of the sampling times used were rather long, and this was done to decrease the data volume. In total, we observed the Crab pulsar for $100 \mathrm{hr}$ at $1.2 \mathrm{GHz}$ and seven hours at $330 \mathrm{MHz}$. The $330 \mathrm{MHz}$ mode was implemented later and used because pulsars, in general, are stronger at lower frequencies and exhibit a steep spectral index (Lorimer et al. 1995). Even though the smaller bandwidth at $330 \mathrm{MHz}$ reduces sensitivity by almost a factor of two, the steep spectrum of the Crab pulsar $(\alpha=3.1 \pm 0.2$ Lorimer et al. 1995, where $S=v^{-\alpha}$ ) means that the pulsar is 55 times brighter at $330 \mathrm{MHz}$ than at $1.2 \mathrm{GHz}$. The flux density from the Crab Nebula is greater at lower frequencies (Cordes et al. 2004) but, due to a shallow power-law index $\left(v^{-0.27}\right)$ (Allen 1973; Bietenholz et al. 1997), at $330 \mathrm{MHz}$ (flux density $\sim 1288 \mathrm{Jy}$ ) is only 1.4 times brighter than at $1.2 \mathrm{GHz}$ (flux density $\sim 909 \mathrm{Jy}$ ). Therefore there should be many more GPs above our $10 \sigma$ threshold at $330 \mathrm{MHz}$ than at $1.2 \mathrm{GHz}$.

Data were taken with WUPPI, the West Virginia University Ultimate Pulsar Processing Instrument. WUPPI is a clone of GUPPI (Green Bank Ultimate Pulsar Processing Instrument; DuPlain et al. 2008) for use with the GB43. GUPPI is a flexible digital backend for the GBT. Like GUPPI, WUPPI is built from reconfigurable off-the-shelf hardware and software available from the CASPER (Center for Astronomy Signal Processing and Electronics Research) group (Parsons et al. 2009). Both GUPPI and WUPPI sample the data with 8 bit precision over bandwidths as large as $800 \mathrm{MHz}$, and are capable of recording all four Stokes parameters. To ease disk space usage, only total intensity (Stokes I) was recorded.

The data were processed using a real-time data reduction pipeline on a 16 processor mini-cluster. The pipeline is comprised of a set of scripts, built from freely available analysis software, ${ }^{9}$ that can reduce a file in real time. First, a file in PSRFITS format (Hotan et al. 2004) has its mean bandpass divided out. Frequency channels that have intensities above the resulting mean are flagged as containing radio frequency interference (RFI) and removed. Then the resulting file is dedispersed at both a dispersion measure (DM) of zero and the DM of the $\mathrm{Crab}$, which is $\sim 56.8 \mathrm{pc} \mathrm{cm}^{-3}$; the exact values for

\footnotetext{
9 http://sigproc.sourceforge.net
} 
Table 1

Observational Parameters Including the MJD the Observation was Taken, the Center Frequency Used, the Length of the Observation, the Sampling Time, the DM Used for Dedispersion, and the Number of GPs Found with S/N $\geqslant 10$

\begin{tabular}{|c|c|c|c|c|c|c|}
\hline MJD & $\begin{array}{l}\text { Frequency } \\
\qquad(\mathrm{MHz})\end{array}$ & $\begin{array}{c}\text { Observation } \\
\text { Length (min) }\end{array}$ & $\begin{array}{l}\text { Sampling } \\
\text { Time }(\mu \mathrm{s})\end{array}$ & $\begin{array}{c}\mathrm{DM} \\
\left(\mathrm{pc} \mathrm{cm}^{-3}\right)\end{array}$ & $\begin{array}{c}\text { Number } \\
\text { of MP GPs }\end{array}$ & $\begin{array}{l}\text { Number } \\
\text { of IP GPs }\end{array}$ \\
\hline 55079 & 1200 & 5 & 204.8 & 56.8005 & 33 & 1 \\
\hline 55079 & 1200 & 108 & 204.8 & 56.8005 & 341 & 27 \\
\hline *55083 & 1200 & 4 & 204.8 & 56.8005 & 19 & 1 \\
\hline *55083 & 1200 & 120 & 204.8 & 56.8005 & 582 & 42 \\
\hline 55085 & 1200 & 120 & 204.8 & 56.8005 & 73 & 0 \\
\hline *55086 & 1200 & 156 & 204.8 & 56.8005 & 1677 & 96 \\
\hline *55090 & 1200 & 150 & 204.8 & 56.8005 & 551 & 31 \\
\hline *55093 & 1200 & 168 & 204.8 & 56.8005 & 650 & 42 \\
\hline *55095 & 1200 & 108 & 51.2 & 56.8005 & 669 & 47 \\
\hline *55096 & 1200 & 150 & 204.8 & 56.8005 & 264 & 21 \\
\hline *55097 & 1200 & 240 & 204.8 & 56.8005 & 519 & 45 \\
\hline *55099 & 1200 & 210 & 204.8 & 56.8005 & 1435 & 76 \\
\hline *55102 & 1200 & 150 & 204.8 & 56.8005 & 1228 & 76 \\
\hline 55118 & 1200 & 6 & 204.8 & 56.8109 & 2 & 0 \\
\hline 55118 & 1200 & 6 & 51.2 & 56.8109 & 16 & 0 \\
\hline 55139 & 1200 & 240 & 51.2 & 56.8229 & 3757 & 308 \\
\hline 55142 & 1200 & 120 & 51.2 & 56.8229 & 244 & 22 \\
\hline 55172 & 1200 & 22 & 51.2 & 56.8279 & 25 & 0 \\
\hline 55178 & 1200 & 282 & 51.2 & 56.8279 & 1808 & 110 \\
\hline 55180 & 1200 & 114 & 51.2 & 56.8279 & 478 & 27 \\
\hline 55240 & 1200 & 7 & 245.76 & 56.8053 & 32 & 2 \\
\hline 55240 & 1200 & 180 & 122.88 & 56.8053 & 1575 & 101 \\
\hline 55256 & 1200 & 15 & 61.44 & 56.8622 & 148 & 8 \\
\hline 55257 & 1200 & 198 & 245.76 & 56.8622 & 1098 & 76 \\
\hline 55261 & 1200 & 492 & 245.76 & 56.8622 & 41 & 9 \\
\hline 55264 & 1200 & 15 & 61.44 & 56.8622 & 106 & 5 \\
\hline 55269 & 1200 & 360 & 245.76 & 56.8622 & 42 & 2 \\
\hline 55270 & 1200 & 30 & 245.76 & 56.8622 & 291 & 17 \\
\hline 55284 & 1200 & 180 & 245.76 & 56.8622 & 1094 & 63 \\
\hline 55290 & 1200 & 108 & 245.76 & 56.8228 & 169 & 10 \\
\hline 55299 & 1200 & 10 & 245.76 & 56.8228 & 4 & 0 \\
\hline 55304 & 1200 & 60 & 245.76 & 56.8228 & 73 & 5 \\
\hline 55346 & 1200 & 60 & 245.76 & 56.8022 & 672 & 40 \\
\hline 55347 & 1200 & 120 & 245.76 & 56.8022 & 1170 & 76 \\
\hline 55347 & 1200 & 168 & 61.44 & 56.8022 & 2701 & 243 \\
\hline 55352 & 1200 & 240 & 61.44 & 56.8022 & 2182 & 146 \\
\hline 55403 & 330 & 37 & 223.42 & 56.7988 & 271 & 31 \\
\hline 55405 & 330 & 60 & 819.2 & 56.7988 & 1201 & 250 \\
\hline 55406 & 330 & 222 & 819.2 & 56.7988 & 1775 & 273 \\
\hline 55411 & 330 & 60 & 819.2 & 56.7962 & 1332 & 220 \\
\hline 55412 & 330 & 30 & 819.2 & 56.7962 & 653 & 114 \\
\hline 55516 & 1200 & 550 & 245.76 & 56.8065 & 4870 & 326 \\
\hline 55532 & 1200 & 315 & 61.44 & 56.7964 & 11671 & 937 \\
\hline 55539 & 1200 & 10 & 61.44 & 56.7964 & 786 & 146 \\
\hline 55541 & 1200 & 335 & 61.44 & 56.7964 & 33611 & 7687 \\
\hline
\end{tabular}

Notes. The starred MJDs are days in which simultaneous observations with the GBT occurred.

each observation, obtained from the Jodrell Bank Crab Pulsar Monthly Ephemeris ${ }^{10}$ (Lyne et al. 1993), are listed in Table 1. The DM of the Crab pulsar can vary on a monthly timescale by $\sim 0.01 \mathrm{pc} \mathrm{cm}^{-3}$. In order to keep processing in real-time we did not correct for this. To ease space requirements, raw data were not kept, so we could also not correct for this later. Errors in GP arrival times due to an incorrect DM on the order of $0.01 \mathrm{pc} \mathrm{cm}^{-3}$ are $\sim 20 \mu \mathrm{s}$, which is less than the sampling time. Both resulting time series are then searched for single pulses with a peak signal-to-noise ratio $(\mathrm{S} / \mathrm{N})$ above $10 \sigma$ us-

\footnotetext{
10 http://www.jb.man.ac.uk/pulsar/crab.html
}

ing our single pulse search (based on the algorithm described by Cordes \& McLaughlin 2003). Our $10 \sigma$ definition of a GP is somewhat arbitrary because there is no set threshold for a GP as the weakest GPs have yet to be observed. The current population of observed GPs accounts for no less than $50 \%$ of the pulsed emission at frequencies around $1.6 \mathrm{GHz}$, and the inclusion of the weakest GPs could bring that number up to $90 \%$ (Majid et al. 2011). A GP time-of-arrival (TOA) (measured by taking the arrival time of the peak of the pulse), the $\mathrm{S} / \mathrm{N}$ of that peak, and the pulse width, taken as the width of a best-fit top hat function, are recorded for each pulse. The TOAs from each time series are compared and if a pulse detected at the DM of the Crab is within $0.1 \mathrm{~s}$ of a zero DM pulse, the $\mathrm{S} / \mathrm{N}$ is checked for both pulses. ${ }^{11}$ If the $\mathrm{S} / \mathrm{N}$ is higher in the zero DM pulse, the pulse is assumed to be due to RFI and removed. The pipeline outputs a profile for each GP and produces an average folded profile for the entire observation, a plot of GP arrival time versus pulse phase, and an average GP profile, made by summing all of the individual GP profiles.

Figure 2 shows the number of GPs detected versus pulse phase. It is worth noting that, although there were only four post-flare observing epochs, there are many more GPs than were recorded in all of the pre-flare data. This was due to a receiver upgrade which resulted in a large increase in sensitivity between our observations on MJD 55412 and MJD 55516. At frequencies below $5 \mathrm{GHz}$ (Cordes et al. 2004), most detected GPs come at the phase of the MP, with the rest at the IP phase. In our data, $\sim 87 \%$ of detected GPs were at the phase of the MP, while the other $\sim 13 \%$ were at the phase of the IP. Only $5 \%$ of GPs detected by Cordes et al. (2004) at $1.2 \mathrm{GHz}$ were at the phase of the IP, but Karuppusamy et al. (2010) found that $\sim 12 \%$ of GPs detected at $1.4 \mathrm{GHz}$ were IP GPs. For detected pulses that show up out of phase with the MP and IP, the frequency versus time plots are checked by eye to see if they show the proper quadratic frequency sweep for a DM of $56.8 \mathrm{pc} \mathrm{cm}^{-3}$. If they do not, those pulses are removed. In most cases, the RFI removal process mentioned above removes most of these false detections before they are checked by eye. We found no events at phases other than those of the MP or IP that showed the proper frequency sweep to be a real GP.

Most of the GPs we detected have a constant intensity across the entire band. Some of them, however, show variations in amplitude as a function of frequency, which have been seen before (e.g., Karuppusamy et al. 2010). We also see variations in amplitude between days, which is likely due to refractive interstellar scintillation (RISS), which can affect the strength of pulses on a timescale of days.

In order to determine if the time stamps for the GB43 are accurate, a short ( $\sim 10 \mathrm{~min})$ observation of the Crab pulsar was made contemporaneously on MJD 55406 at similar frequencies with the GB43 and GBT. The GB43 used a center frequency of $330 \mathrm{MHz}$, while the GBT used $350 \mathrm{MHz}$. GPs were detected in each data set and their TOAs were compared. As can be seen in Figure 3, the pulse shapes of contemporaneous GPs are similar, and the TOAs, converted to infinite frequency at the solar system barycenter, have identical arrival times to within the instrumental resolution.

Figure 4 shows the average profile of the Crab pulsar at 1.2 $\mathrm{GHz}$ and $330 \mathrm{MHz}$. The intensities are in arbitrary units. The MP and IP are visible at both frequencies. The increased

\footnotetext{
11 This is of the order of the frequency dependent arrival time delay across the band, and is used as it removes very nearly all RFI, while removing few real GPs.
} 


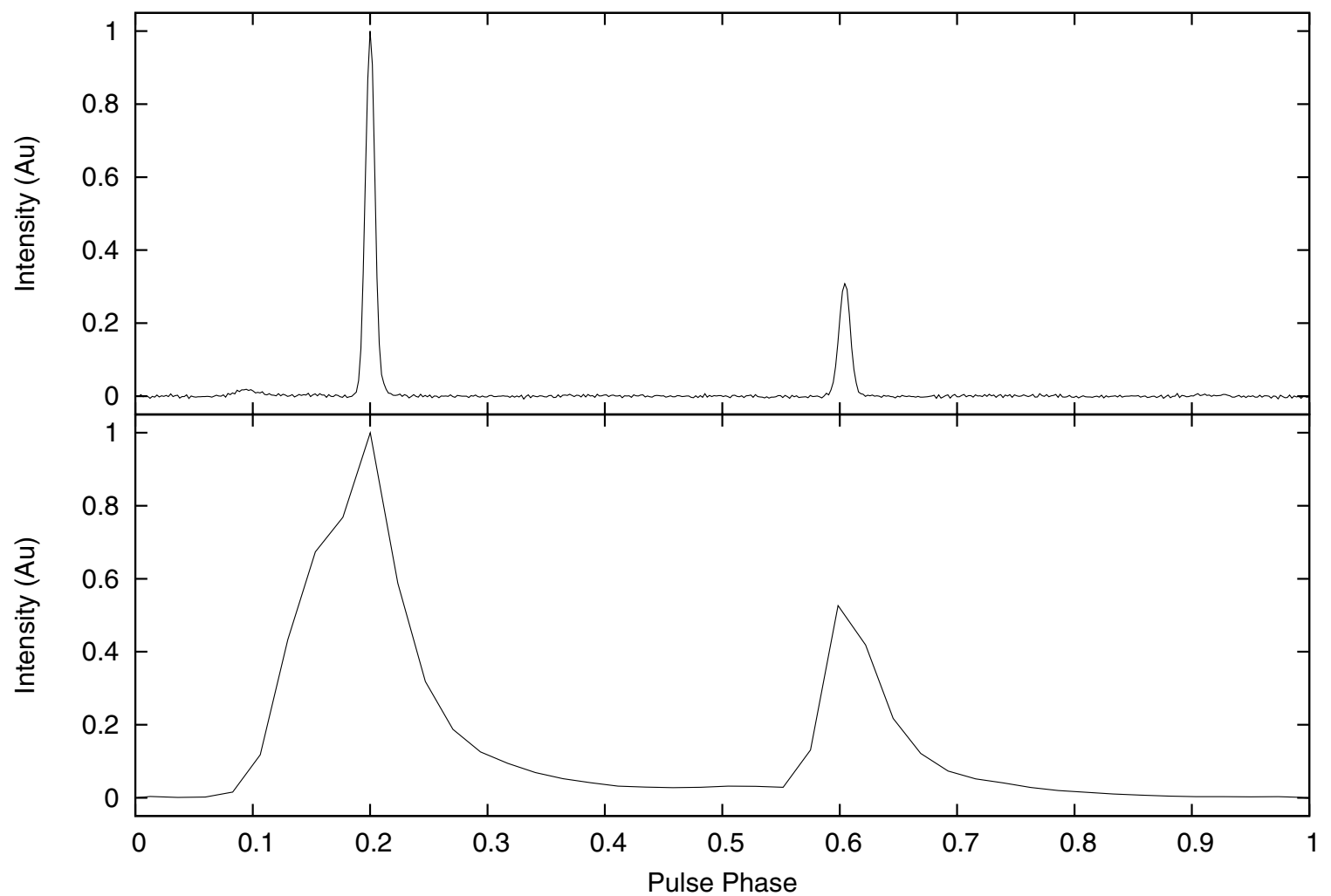

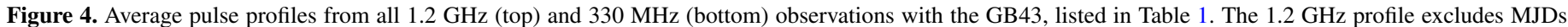

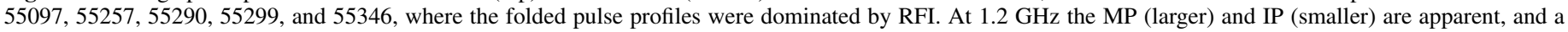

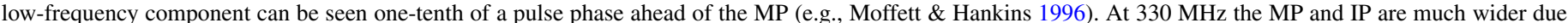

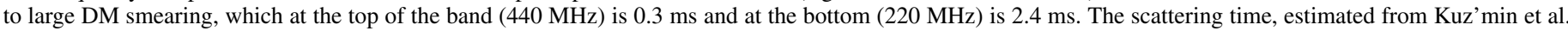

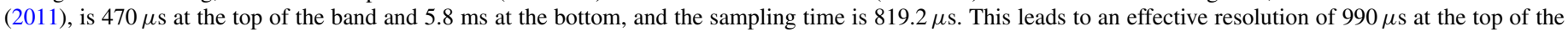
band and $6.3 \mathrm{~ms}$ at the bottom.

Table 2

Sampling Times, DM Smearing Times, Scattering Times, and Effective Resolution at the Top and Bottom of the Band for the GB43 $330 \mathrm{MHz}$ and 1.2 GHz Observations, as well as for the GBT $350 \mathrm{MHz}$ Observation

\begin{tabular}{|c|c|c|c|c|c|c|c|}
\hline \multirow{2}{*}{$\begin{array}{l}\text { Frequency } \\
(\mathrm{MHz})\end{array}$} & \multirow{2}{*}{$\begin{array}{c}\text { Sampling Time } \\
\text { (us) }\end{array}$} & \multicolumn{2}{|c|}{ DM Smearing Time } & \multicolumn{2}{|c|}{ Scattering Time } & \multicolumn{2}{|c|}{ Effective Resolution } \\
\hline & & Top & Bottom & Top & Bottom & Top & Bottom \\
\hline 330 (GB43) & 819.2 & $300 \mu \mathrm{s}$ & $2.4 \mathrm{~ms}$ & $470 \mu \mathrm{s}$ & $5.8 \mathrm{~ms}$ & $990 \mu \mathrm{s}$ & $6.3 \mathrm{~ms}$ \\
\hline 350 (GBT) & 81.92 & $361 \mu \mathrm{s}$ & $855 \mu \mathrm{s}$ & $670 \mu \mathrm{s}$ & $1.9 \mathrm{~ms}$ & $765 \mu \mathrm{s}$ & $2.0 \mathrm{~ms}$ \\
\hline $1200(\mathrm{~GB} 43)$ & 204.8 & $23 \mu \mathrm{s}$ & $180 \mu \mathrm{s}$ & $4 \mu \mathrm{s}$ & $54 \mu \mathrm{s}$ & $206 \mu \mathrm{s}$ & $278 \mu \mathrm{s}$ \\
\hline
\end{tabular}

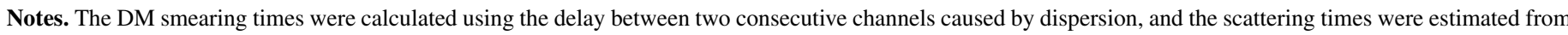
Kuz'min et al. (2011). The sampling time listed for the $1.2 \mathrm{GHz}$ GB43 observation was the most common sampling time from Table 1.

width at low frequency is due largely to DM smearing, which is on the order of $2.4 \mathrm{~ms}$ at the bottom of the band. The $1.2 \mathrm{GHz}$ profile exhibits a weak low-frequency component (LFC), about one-tenth of a pulse phase ahead of the MP (e.g., Moffett \& Hankins 1996). At $330 \mathrm{MHz}$ there is a precursor (PR) to the MP (e.g., Rankin et al. 1970) which is broad enough at this frequency that it shows up as the first peak of the double-peaked MP (see Karuppusamy et al. 2012, for the frequency evolution of the PR), which is visible in the low-frequency GBT profile from MJD 55406 (Figure 1). Due to a much larger effective resolution, the MP in the GB43 profile is unresolved and appears to have a single peak. Table 2 lists the effective resolutions at the top and bottom of the band for both the $330 \mathrm{MHz}$ and $1.2 \mathrm{GHz}$ GB43 observations as well as the GBT $350 \mathrm{MHz}$ observation.

The full widths at half maximum (FWHMs) of the MP and IP from the GB43 profiles are $\sim 305 \mu$ s and $\sim 360 \mu$ s at $1.2 \mathrm{GHz}$ and $\sim 3.2 \mathrm{~ms}$ and $\sim 1.5 \mathrm{~ms}$ at $330 \mathrm{MHz}$. At $330 \mathrm{MHz}$, the $\mathrm{MP}$ is wider than the IP due to its overlap with the PR (Rankin et al.
1970). The average GP profiles, however, are narrower than the average folded profiles at both frequencies (see Figure 5), with FWHMs of $\sim 140 \mu$ s for both the MP and IP at $1.2 \mathrm{GHz}$, and $\sim 1.1 \mathrm{~ms}$ at $330 \mathrm{MHz}$ for both the MP and IP. It has been suggested by Popov et al. (2006) that every pulse at the phase of the MP and IP is a GP, and that normal emission only comes from the PR, where no GPs have been seen. We also have not seen any GPs at the phase of the PR at $330 \mathrm{MHz}$ in our data. Even though our observed GP profiles are narrower than the average folded profiles, this hypothesis is not ruled out. Popov \& Stappers (2007) found that pulse width is inversely proportional to pulse intensity, so lowering our $\mathrm{S} / \mathrm{N}$ definition of a GP would include wider GPs which could possibly increase the width of the average GP profile to that of the folded profile. Also, the phases of weaker GPs may have more deviation from the center phase of the MP and IP than stronger GPs, leading to a wider average profile. Unfortunately, we were unable to lower our GP threshold below $10 \sigma$ as this resulted in many spurious pulses. 


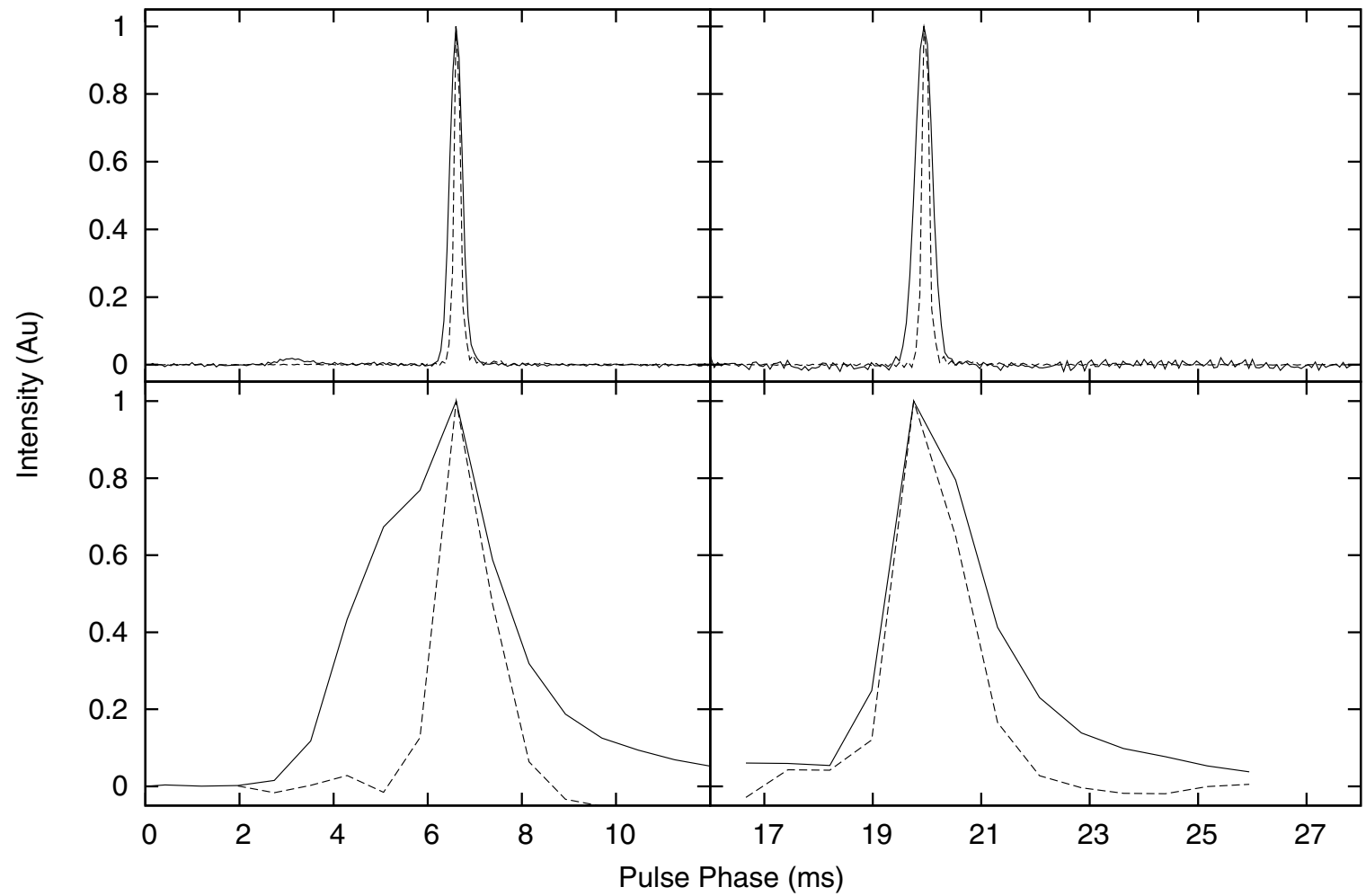

Figure 5. Average GP profiles for all MP (left) and IP (right) GPs (dashed lines) collected with the GB43 at $1.2 \mathrm{GHz}$ (top) and $330 \mathrm{MHz}$ (bottom). The $1.2 \mathrm{GHz}$ profiles are made by summing the 76707 individual MP and 10871 individual IP GP profiles, and the $330 \mathrm{MHz}$ profiles are made by summing the 5232 individual MP and 888 individual IP GP profiles. They are shown with the folded MP and IP profiles for all $1.2 \mathrm{GHz}$ and $330 \mathrm{MHz}$ data (solid lines). The FWHM of the $1.2 \mathrm{GHz}$ folded MP and IP are $\sim 305 \mu$ s and $\sim 360 \mu$ s, respectively, while the FWHM of the average MP and IP GPs are both $\sim 140 \mu$ s. The FWHM of the 330 MHz folded MP and IP are $\sim 3.2 \mathrm{~ms}$ and $\sim 1.5 \mathrm{~ms}$, respectively, while the FWHM of the average MP and IP GPs are $\sim 1.1 \mathrm{~ms}$. The $330 \mathrm{MHz}$ MP is much wider than the IP due to its overlap with the MP precursor. The intensities of the profiles are arbitrary and are scaled for clarity.

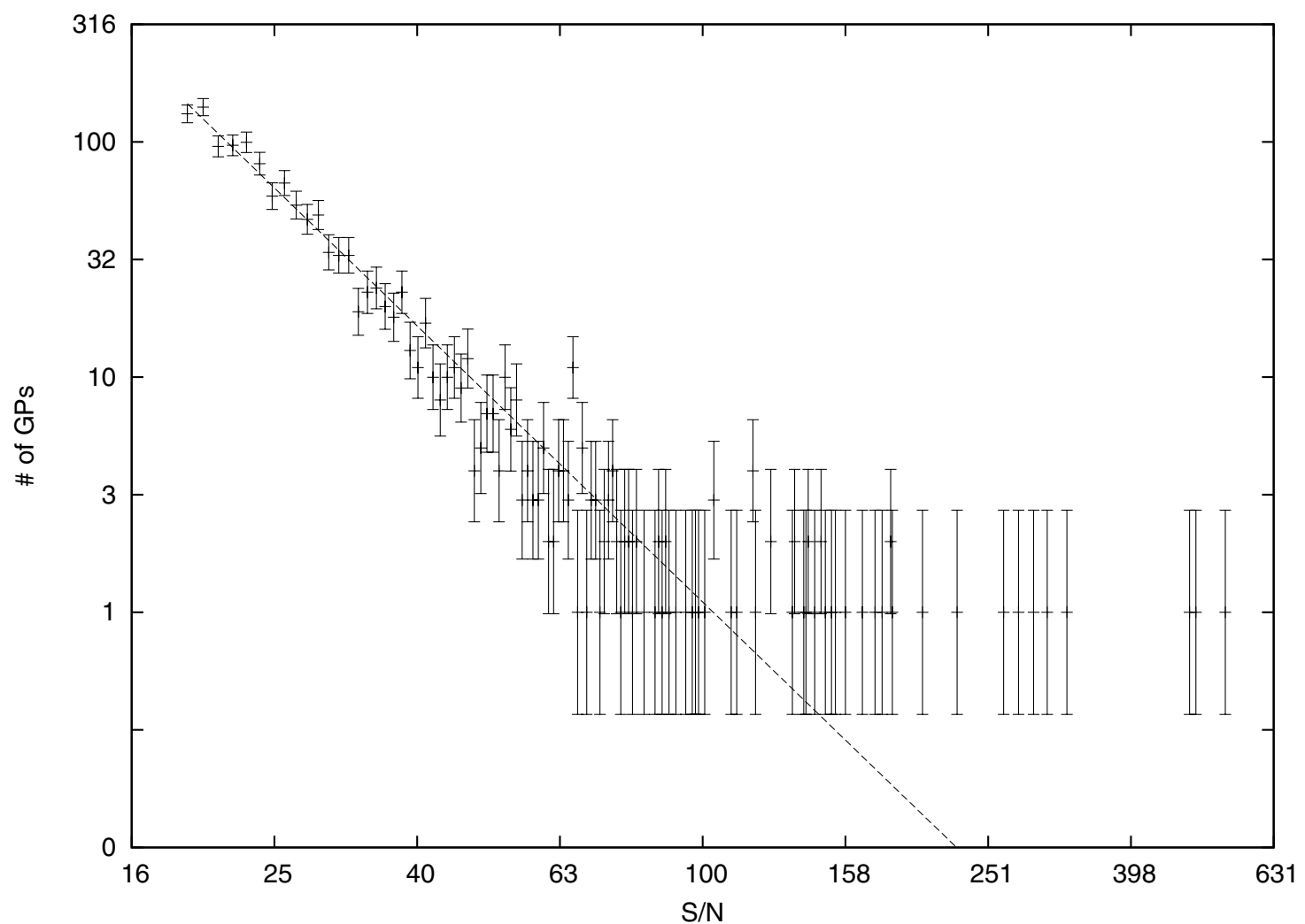

Figure 6. Plot of the log of the number of GPs collected with the GB43 vs. the log of their S/N for the MP at $1.2 \mathrm{GHz}$ on MJD 55099 and a best-fit power law, which has a slope of $2.93 \pm 0.07$. The deviation from the power-law distribution at high $\mathrm{S} / \mathrm{N}$ is seen on all days, and these GPs may be examples of supergiant pulses, seen previously by Cordes et al. (2004). The average supergiant pulse is about 15 times stronger than the average GP. 
Table 3

Differential Power-law Measurements for the MP and IP for each Observing Epoch

\begin{tabular}{|c|c|c|}
\hline MJD & $\begin{array}{l}\text { Differential Power- } \\
\text { law Index (MP) }\end{array}$ & $\begin{array}{l}\text { Differential Power- } \\
\text { law Index (IP) }\end{array}$ \\
\hline 55079 & $\mathrm{X}$ & $\mathrm{X}$ \\
\hline 55079 & $2.1 \pm 0.3$ & $X$ \\
\hline 55083 & $\mathrm{X}$ & $X$ \\
\hline 55083 & $3.1 \pm 0.2$ & $\mathrm{X}$ \\
\hline 55085 & X & $X$ \\
\hline 55086 & $2.70 \pm 0.06$ & $X$ \\
\hline 55090 & $2.8 \pm 0.1$ & $X$ \\
\hline 55093 & $2.6 \pm 0.2$ & $X$ \\
\hline 55095 & $2.9 \pm 0.2$ & $X$ \\
\hline 55096 & $X$ & $X$ \\
\hline 55097 & $\mathrm{X}$ & $X$ \\
\hline 55099 & $2.93 \pm 0.07$ & $\mathrm{X}$ \\
\hline 55102 & $2.92 \pm 0.08$ & $X$ \\
\hline 55118 & $\mathrm{X}$ & $\mathrm{X}$ \\
\hline 55118 & $\mathrm{X}$ & $X$ \\
\hline 55139 & $2.61 \pm 0.08$ & $\mathrm{X}$ \\
\hline 55142 & $\mathrm{X}$ & $X$ \\
\hline 55172 & $\mathrm{X}$ & $X$ \\
\hline 55178 & $2.81 \pm 0.08$ & $X$ \\
\hline 55180 & $\mathrm{X}$ & $X$ \\
\hline 55240 & $\mathrm{X}$ & $X$ \\
\hline 55240 & $2.1 \pm 0.1$ & $\mathrm{X}$ \\
\hline 55256 & $\mathrm{X}$ & $X$ \\
\hline 55257 & $2.1 \pm 0.1$ & $X$ \\
\hline 55261 & $\mathrm{X}$ & $X$ \\
\hline 55264 & $\mathrm{X}$ & $X$ \\
\hline 55269 & $\mathrm{X}$ & $X$ \\
\hline 55270 & $\mathrm{X}$ & $X$ \\
\hline 55284 & $2.1 \pm 0.1$ & $X$ \\
\hline 55290 & $\mathrm{X}$ & $X$ \\
\hline 55299 & $\mathrm{X}$ & $X$ \\
\hline 55304 & $\mathrm{X}$ & $X$ \\
\hline 55346 & $2.4 \pm 0.2$ & $X$ \\
\hline 55347 & $2.8 \pm 0.1$ & $\mathrm{X}$ \\
\hline 55347 & $2.40 \pm 0.07$ & $X$ \\
\hline 55352 & $2.21 \pm 0.07$ & $X$ \\
\hline 55403 & $2.5 \pm 0.2$ & $X$ \\
\hline 55405 & $2.95 \pm 0.09$ & $2.4 \pm 0.2$ \\
\hline 55406 & $2.94 \pm 0.05$ & $3.1 \pm 0.2$ \\
\hline 55411 & $2.8 \pm 0.1$ & $2.9 \pm 0.2$ \\
\hline 55412 & $2.7 \pm 0.2$ & $X$ \\
\hline 55516 & $2.93 \pm 0.05$ & $2.4 \pm 0.4$ \\
\hline 55532 & $2.56 \pm 0.05$ & $2.7 \pm 0.2$ \\
\hline 55541 & $2.8 \pm 0.1$ & $2.81 \pm 0.03$ \\
\hline
\end{tabular}

Notes. Some MJDs have two entries listed because there were two separate observations on those days. An X means that there were not enough pulses recorded for the MP or IP on that day to fit a power law to the amplitude distribution.

\section{AMPLITUDE DISTRIBUTIONS}

A total of 93,698 GPs were observed with the GB43. At $1.2 \mathrm{GHz}$ we recorded 76,707 GPs at the phase of the MP and $10,871 \mathrm{GPs}$ at the phase of the IP, and at $330 \mathrm{MHz} 5232 \mathrm{MP}$ and 888 IP GPs were recorded. Due to low GP statistics on some days, only 78,574 MP GPs and 9693 IP GPs were used in fitting the amplitude distributions.

We calculated, through least-squares fitting, power-law indices for the differential amplitude distributions of MP and IP GPs at both $1.2 \mathrm{GHz}$ and $330 \mathrm{MHz}$ for each day separately.
Figure 6 is an example of a power-law fit for the MP. We noted that the power-law index of GPs varied daily, as was previously reported by Lundgren et al. (1995) at similar frequencies. This gave a range of power-law indices from $2.1 \pm 0.3$ to $3.1 \pm 0.2$ for MP GPs at $1.2 \mathrm{GHz}, 2.4 \pm 0.4$ to $2.81 \pm 0.03$ for IP GPs at $1.2 \mathrm{GHz}, 2.5 \pm 0.2$ to $2.95 \pm 0.09$ for MP GPs at $330 \mathrm{MHz}$, and $2.4 \pm 0.2$ to $3.1 \pm 0.2$ for IP GPs at $330 \mathrm{MHz}$. Table 3 lists the power-law indices for each day. These indices agree with other published power-law indices, listed in Table 4. The range of indices is likely due to wider GPs having steeper spectra, as was seen by Popov et al. (2008). We did not have the time resolution to separate the GPs by width, as the widest GPs reported by Popov et al. (2008) were $64 \mu \mathrm{s}$, which is the shortest sampling time we used.

We were unable to properly calibrate the data due to lack of any off-source pointings and/or observations with a pulsed cal. There were also doubts as to the stability of the receiver over long timespans. We were able to do a rough calibration, however, by correcting for RISS, which affects the strength of all GPs by the same amount, so there should be no change to the power-law slope. Rickett \& Lyne (1990) found that the RISS timescale for the Crab pulsar scales as $v^{-2.2}$. Based on this, the timescales at $1.2 \mathrm{GHz}$ and $330 \mathrm{MHz}$ are 1.7 days and 30 days, respectively. A Lomb-Scargle analysis (Scargle 1982) of the periodicity of GP arrival times yielded periodicities of 0.41 days at $330 \mathrm{MHz}$ and 0.99 days at $1.2 \mathrm{GHz}$. The $\sim 1$ day periodicity of the GP arrival times in the $1.2 \mathrm{GHz}$ data is on the order of the RISS timescale at that frequency, but the same periodicities were also seen in the randomized data set, so they are not significant. We attempted to correct for RISS by scaling observations based on the brightness of their folded profiles. However, if GPs dominate the average folded profile, as was seen by Popov et al. (2006), any intrinsic variations in their strength will affect this correction. To determine if this was true, we removed the GPs from the average profile. We found that at $1.2 \mathrm{GHz}$ the amplitude of the average profile without GPs was $1 \%$ less than the average profile with the GPs, and at $330 \mathrm{MHz}$ was $2 \%$ less. We therefore concluded that GPs do not dominate the average profile. One possible reason why our results are the opposite of what was seen by Popov et al. (2006) is that they recorded all GPs above $5 \sigma$, while we were only able to record GPs above $10 \sigma$. Therefore, it is possible that weaker GPs dominate the average profile. If the average profile is truly dominated by GPs, then correcting for RISS is complicated, but since we do not see this, we used the following correction.

We took the brightest folded profile from each of our three observing epochs (pre-flare $1.2 \mathrm{GHz}, 330 \mathrm{MHz}$, post-flare 1.2 $\mathrm{GHz}$ ) and scaled all of the folded profiles from those epochs to that profile. That provided us with a scaling factor for each day, by which we then multiplied the GP S/Ns. We also compared our indices with power-law indices of other source classes in an attempt to constrain the GP emission physics. Other source classes and their indices are listed in Table 5. Although most normal pulsars have log-normal distributions, some pulsars have amplitude distributions that have power-law tails. These power-law exponents are included in Table 5 under "Normal Pulsars." Our power-law indices match those of magnetars and RRATs, but do not match the power-law tails seen from normal pulsars.

Also seen in Figure 6 is a non power-law tail, which is seen on all days in both the MP and IP. This deviation is significant, and was also seen by Cordes et al. (2004), who postulated these outlying GPs could be supergiant pulses, indicating that 
Table 4

Comparison of Differential Power-law Indices for the MP and IP at Both 1.2 GHz and $330 \mathrm{MHz}$ between This Work and Previously Published Values

\begin{tabular}{|c|c|c|c|}
\hline $\begin{array}{l}\text { Frequency } \\
(\mathrm{MHz})\end{array}$ & $\begin{array}{c}\text { Differential Power- } \\
\text { law Index (MP) }\end{array}$ & $\begin{array}{l}\text { Differential Power- } \\
\text { law Index (IP) }\end{array}$ & Reference \\
\hline 112 & $3.3^{\mathrm{a}}$ & $\ldots$ & Smirnova \& Logvinenko (2009) \\
\hline 146 & 3.5 & 3.8 & Argyle \& Gower (1972) \\
\hline 200 & $2.7^{\mathrm{a}}$ & $\ldots$ & Bhat et al. (2007) \\
\hline 330 & $2.5-3.0$ & $2.4-3.1$ & This work \\
\hline 430 & 2.3 & $\ldots{ }^{b}$ & Cordes et al. (2004) \\
\hline 600 & 3.2 & 3.0 & Popov et al. (2009) \\
\hline 812 & $3.3^{\mathrm{a}}$ & $\ldots$ & Lundgren et al. (1995) \\
\hline 1200 & $2.7-4.2$ & 2.6 & Popov \& Stappers (2007) \\
\hline 1200 & $2.1-3.1$ & $2.4-2.8$ & This work \\
\hline 1300 & $2.3^{\mathrm{a}}$ & $\ldots$ & Bhat et al. (2008) \\
\hline 1400 & 2.8 & 3.1 & Karuppusamy et al. (2010) \\
\hline 2100 & $3.0^{\mathrm{a}}$ & $\ldots$ & Zhuravlev et al. (2011) \\
\hline 4850 & $2.8^{\mathrm{a}}$ & $\ldots$ & Popov et al. (2008) \\
\hline
\end{tabular}

Notes.

${ }^{a}$ MP and IP GPs were combined in these analyses.

b No measurement was taken for the IP.

Table 5

Differential Power-law Indices for Different Source Classes for Comparison with GP Power Laws

\begin{tabular}{lcl}
\hline \hline $\begin{array}{l}\text { Source } \\
\text { Class }\end{array}$ & $\begin{array}{c}\text { Power Law } \\
\text { Index }\end{array}$ & \\
\hline Normal Pulsars & 3.85 & Reference \\
Magnetars & $2.1-7.7$ & Serylak et al. (2009) \\
RRATs & 3.0 & J. J. Miller et al. (2012, in preparation) \\
\hline
\end{tabular}

there may be two distinct mechanisms for GP generation. These supergiant pulses account for slightly less than one percent of the GPs used in the amplitude distributions.

\section{CORRELATING RADIO GPS FROM THE GB43 AND GBT}

For $16 \mathrm{hr}$ over the span of eight days, we observed the Crab pulsar simultaneously with the GB43 and GBT. The center frequency for GB43 observations was $1.2 \mathrm{GHz}$ with $400 \mathrm{MHz}$ of useable bandwidth, while that of the GBT was $8.9 \mathrm{GHz}$ with $800 \mathrm{MHz}$ of useable bandwidth. At high frequencies the majority of GPs come at the phase of the IP, with fewer MP GPs and few GPs from high-frequency components (HFCs; see Figure 7 for a comparison of the folded profile at high and low frequencies). For this work, we did not use any HFC GPs in our correlation analysis. We matched the barycentered arrival times of the 39,900 GPs with $\mathrm{S} / \mathrm{N}>10$ recorded with the GBT (1035 MP GPs, 38,865 IP GPs) with the 7933 GPs recorded simultaneously with the GB43 (7466 MP GPs, 467 IP GPs). We found that 236 low-frequency MP GPs were also detected simultaneously at $8.9 \mathrm{GHz}$. These GPs were neither the strongest nor the weakest pulses from either data set. The chance probability of this occurring is zero percent. This is not surprising, as we know that the high- and low-frequency MP are the same component. We did expect, however, that all of the MP GPs detected at $8.9 \mathrm{GHz}$ would be the strongest of the MP GPs detected at $1.2 \mathrm{GHz}$, since the MP is significantly weaker at $8.9 \mathrm{GHz}$ than at $1.2 \mathrm{GHz}$. All chance probabilities were calculated assuming Poisson statistics, using the formula
$P=\left(e^{\lambda} \times \lambda^{K}\right) / K$ !, where $\lambda$ is the number of detections expected and $K$ is the number of detections actually recorded.

Moffett \& Hankins (1996) first noticed that the low- and highfrequency IP components of the folded profile are separated by $10^{\circ}$, which is about $970 \mu \mathrm{s}$. We checked to see if any lowfrequency IP GPs were simultaneously detected at $8.9 \mathrm{GHz}$. Only 23 were found $(\sim 5 \%)$, with a zero percent chance occurrence, but this is expected since the spectral index of GPs is steep (Popov et al. 2008). We then checked to see if low- and high-frequency IP GPs commonly occurred within the same rotation period of the pulsar, as was previously seen by Popov et al. (2008). In our data, we found 15 instances $(\sim 4 \%)$ of low-frequency IP GPs occurring within one spin period of a high-frequency IP GP. The probability of this measurement happening by chance is $9 \%$. In four of these instances the GPs are within one $\mu$ s of each other and the chance probability of this occurring is zero. In the other 11 instances, the GPs are almost one spin period apart. These are likely statistical, as there is a $10 \%$ chance of this occurring randomly. The fact that only four of the $1.2 \mathrm{GHz}$ IP GPs occur within one $\mu$ s of an $8.9 \mathrm{GHz}$ IP GP suggests that the high and low-frequency IP may be created by different physical processes (Moffett 1997) possibly due to emission from different regions in the magnetosphere (Hankins \& Eilek 2007). However, since the chance probability of detecting four low- and high-frequency IP GPs within one $\mu \mathrm{s}$ is zero, it seems likely that the high-frequency IP is related to the low-frequency IP, as seen by Popov et al. (2008) in previous correlations of IP GPs at 600 and $4850 \mathrm{MHz}$. One possibility is that both the high and low-frequency IP are reflections of the MP off of the magnetosphere (Petrova 2009). In this scenario, radio emission from the MP originating deep in the magnetosphere propagates through the electron-positron plasma that fills the magnetosphere. Transverse scattering causes the MP emission to be backscattered, causing it to arrive at a different pulse phase. If this was the case, we would expect a linear relationship between the strength of the MP and IP in the folded profile for each observation. As shown in Figure 8, we do see such a relationship at both $1.2 \mathrm{GHz}$ and $330 \mathrm{MHz}$. The slopes for the $1.2 \mathrm{GHz}$ and $330 \mathrm{MHz}$ relations are $0.310 \pm 0.004$ and $0.58 \pm$ 0.08 , respectively. 


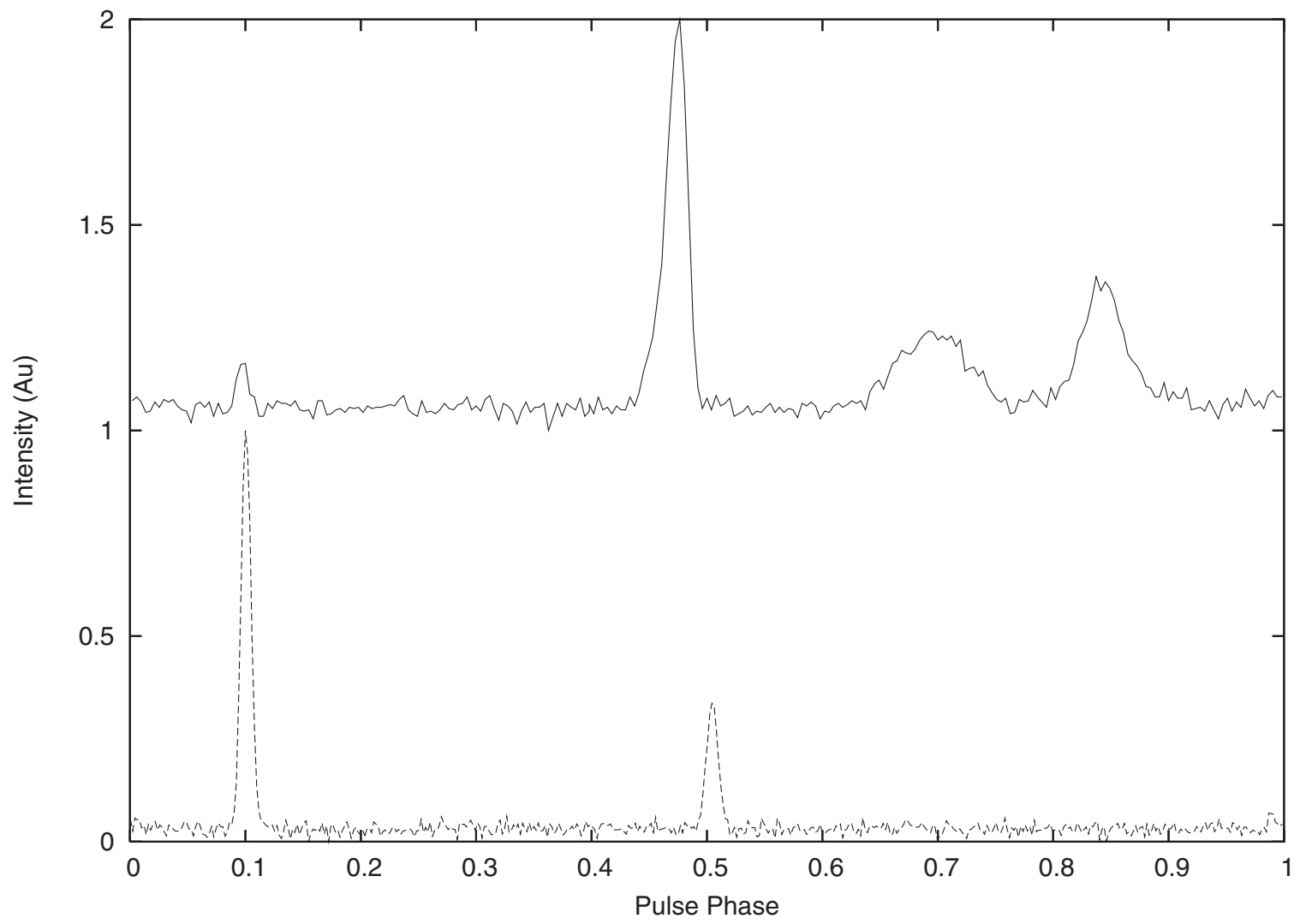

Figure 7. Average profiles from the GBT at $8.9 \mathrm{GHz}$ (solid line; from Bilous et al. 2011) and the GB43 at $1.2 \mathrm{GHz}$ (dashed line). The weakening of the MP at high frequencies can be seen, as well as the strengthening and shift of the IP.

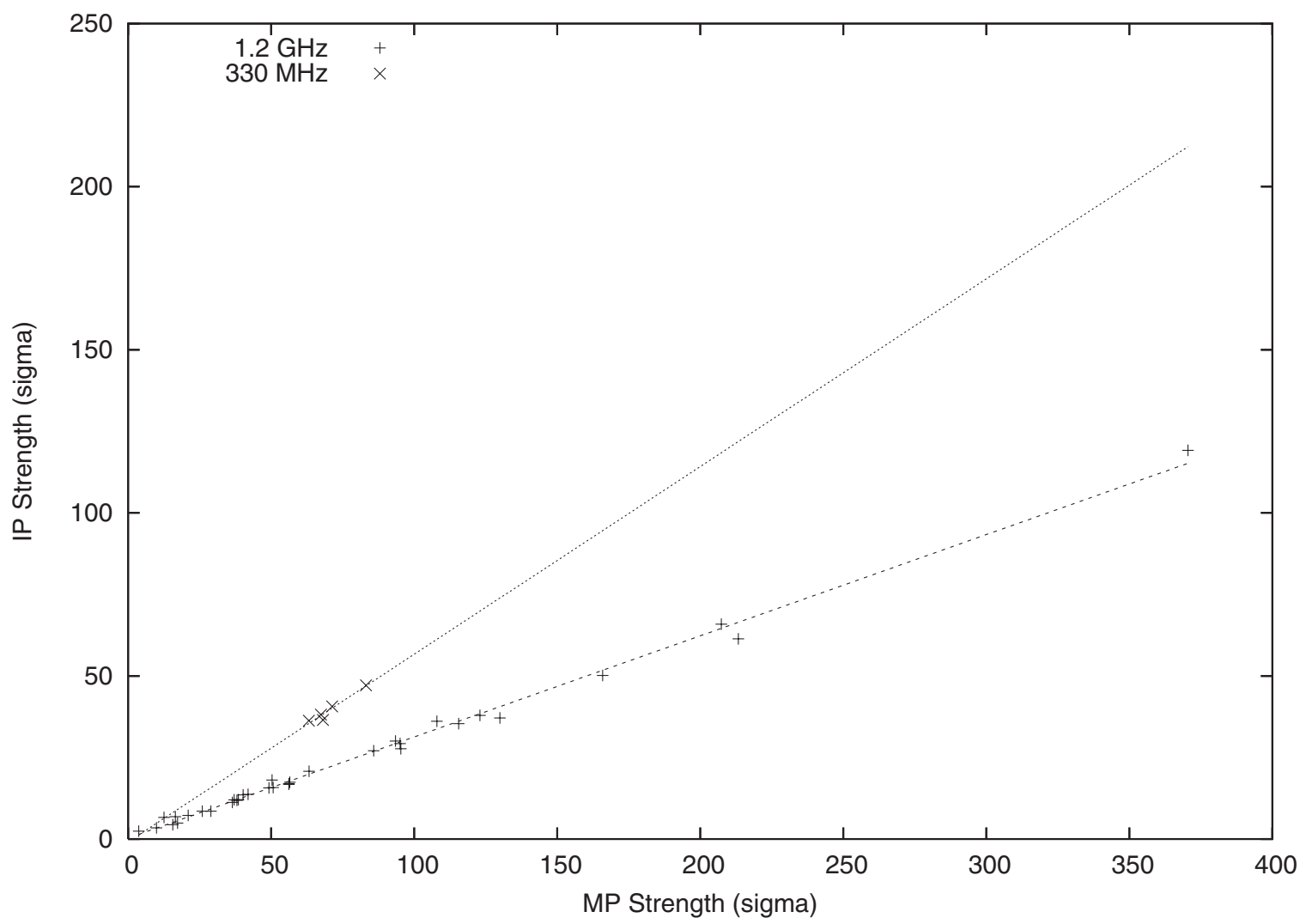

Figure 8. Comparison of the strength of the MP of the folded profile to that of the IP for each observation. There is an obvious linear trend in both the $330 \mathrm{MHz}$ and $1.2 \mathrm{GHz}$ data, with slopes of $0.58 \pm 0.08$ and $0.310 \pm 0.004$, respectively. This supports the theory that the IP may be a reflection of the MP off of the magnetosphere. 


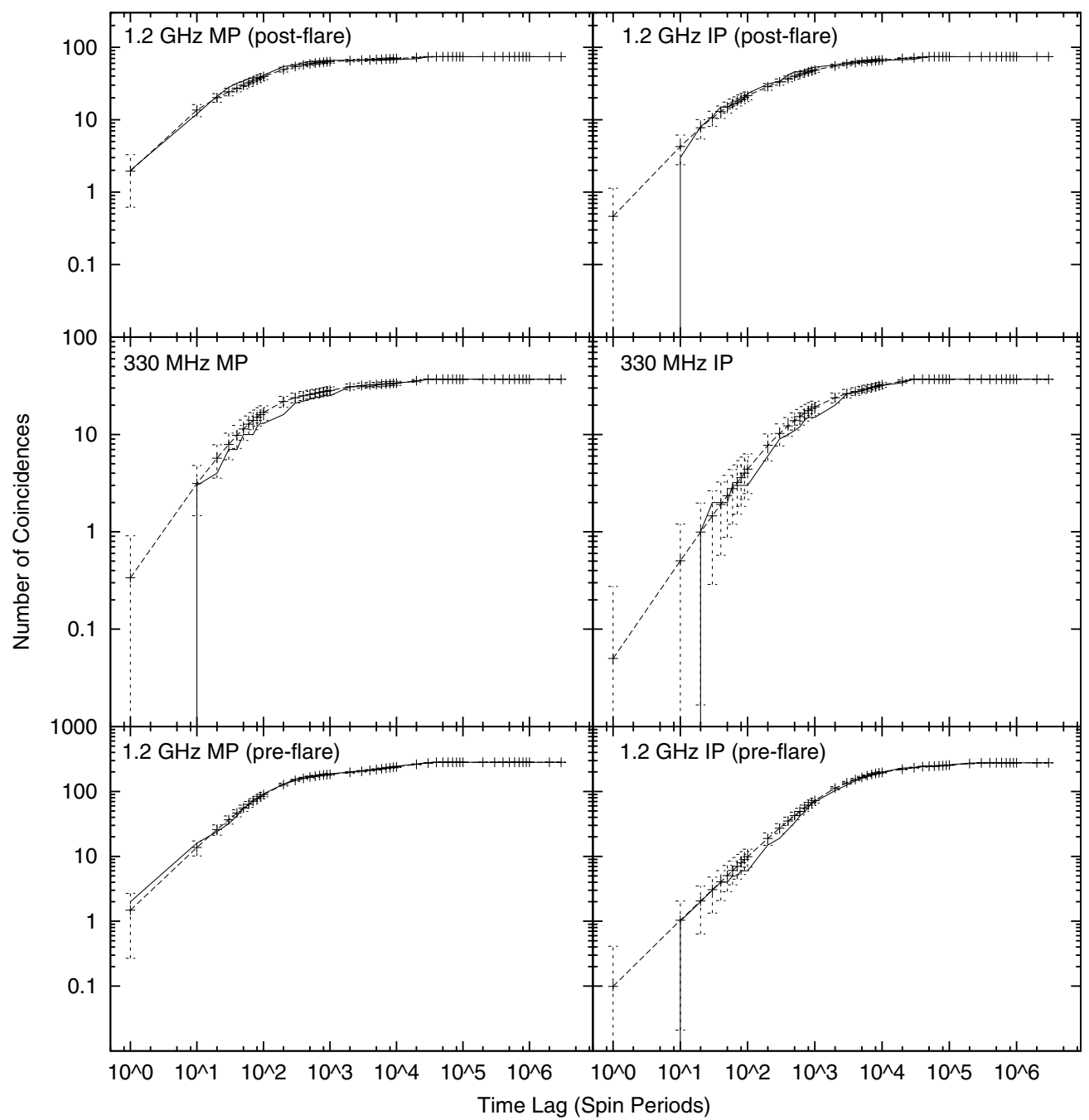

Figure 9. Number of coincidences between Fermi photons and GB43 GP TOAs (solid lines), as well as the number of coincidences between randomized Fermi photons and GP TOAs (dotted line) with $1 \sigma$ error bars. The bottom row is the correlation with the $1.2 \mathrm{GHz}$ pre-flare data, the middle row is the $330 \mathrm{MHz}$ data, and the top row is the $1.2 \mathrm{GHz}$ post-flare data. For each row the MP is the left panel and the IP is the right panel.

\section{CORRELATING RADIO GPs FROM THE GB43 WITH $\gamma$-RAY PHOTONS FROM FERMI}

One of the predictions of Lyutikov (2007) is that there would be increased $\gamma$-ray flux during a GP. In order for this model to accurately reproduce the data, the plasma density of the GP emission region must be $\sim 10^{5}$ higher than the minimum Goldreich-Julian density and the duty cycle of the pulsar must be 0.001 . This increase in density could be due to enhanced pair production in the pulsar magnetosphere. If correct, these high-energy particles, produced during reconnection close to the Y point, where the last closed magnetic field lines approach the light cylinder at the magnetic equator (Lyutikov 2007), are expected to produce curvature radiation from 0.1 to $100 \mathrm{GeV}$ (depending on the value of the Lorentz factor $\gamma$ ) at the time of a GP. This curvature radiation would cause an increase in $\gamma$-rays at the times of radio GPs.

Data were downloaded from the Fermi online archive ${ }^{12}$ for days when radio observations occurred. "Source" class events from Pass 7 data above $100 \mathrm{MeV}$ were selected in an energy dependent radius $(\theta<\operatorname{Max}(6.68-1.76 \log (E), 1.3)$, where $E$ is the energy of the photon in $\mathrm{MeV}$ ) around the position of the

\footnotetext{
12 http://fermi.gsfc.nasa.gov/cgi-bin/ssc/LAT/LATDataQuery.cgi
}

pulsar (Abdo et al. 2010). Only photons in Good Time Intervals (GTIs) were selected and those with a zenith angle $>100^{\circ}$ were excluded to discriminate against $\gamma$-rays generated in Earth's atmosphere. The photons were converted to infinite frequency at the solar system barycenter using the gtbary utility from the Fermi Science Tools package.

We then searched for coincidences between radio GPs and $\gamma$-ray photons. Due to clock errors in the backend on four days at $1.2 \mathrm{GHz}$ (MJDs 55097, 55257, 55290, 55346), only 75,131 MP and 10771 IP GPs were used. However, all of the 5232 MP and 888 IP GPs recorded at $330 \mathrm{MHz}$ were used. This resulted in a total of 92,022 GPs and $393 \gamma$-ray photons, with an average $\gamma$-ray photon rate of $\sim 16$ photons $\mathrm{hr}^{-1}$, in agreement with the 15 photons $\mathrm{hr}^{-1}$ seen by Abdo et al. (2010). Fermi observes the Crab pulsar for about $11 \mathrm{hr}$ per day, resulting in about $34 \mathrm{hr}$ of simultaneous observing time. We also made energy cuts and searched for coincidences between radio GPs and $\gamma$-ray photons above both $500 \mathrm{MeV}$ and $1 \mathrm{GeV}$.

Based on comparisons with correlations of randomized data, we found no significant correlation between MP or IP GPs at both $1.2 \mathrm{GHz}$ and $330 \mathrm{MHz}$ and $\gamma$-ray photons out to a time lag of $\pm 3 \times 10^{6}$ spin periods. Since there was not necessarily the same number of randomized $\gamma$-ray photons 


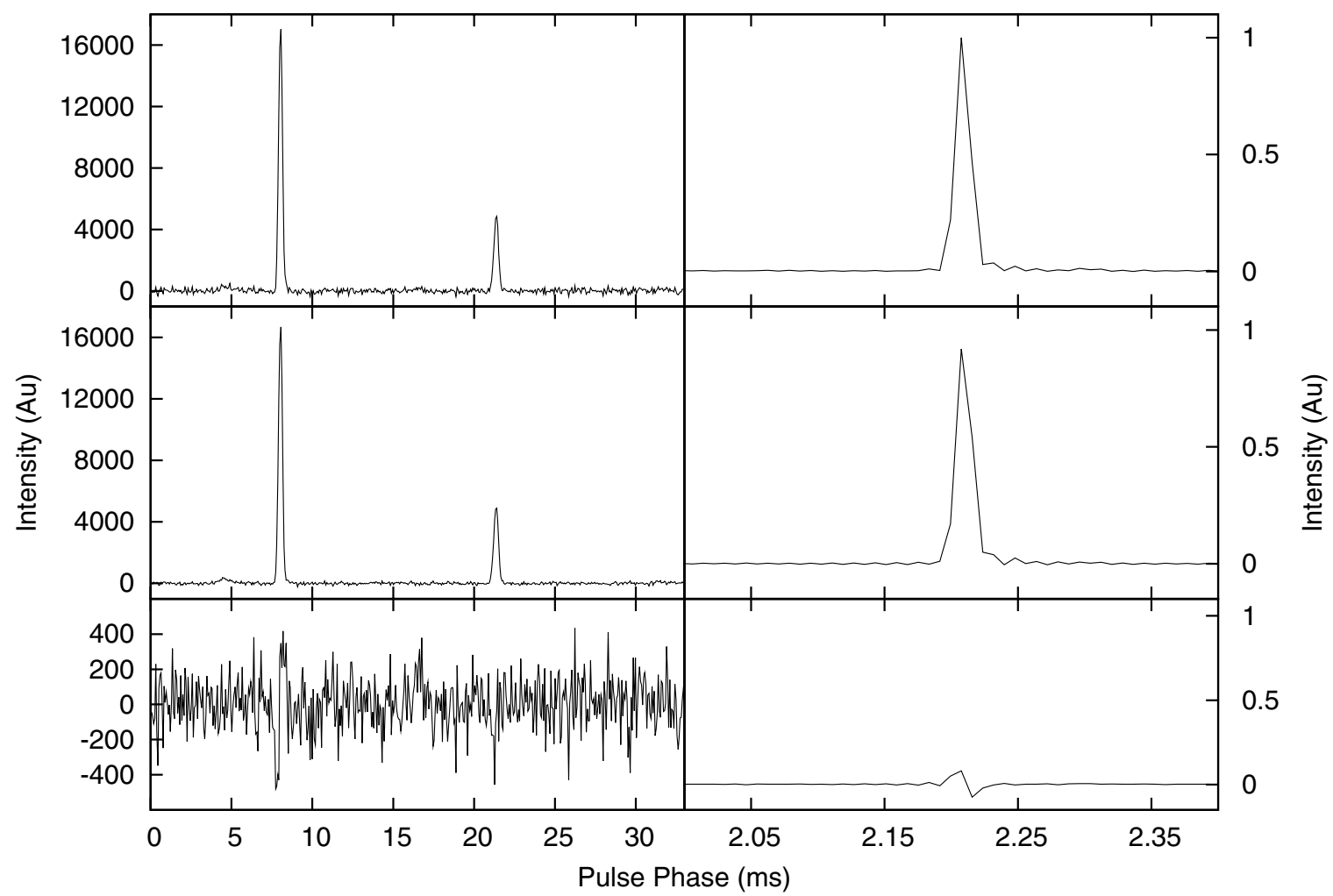

Figure 10. $\gamma$-ray flare occurred from MJDs 55457-55461, so we checked for changes in the average pulse shape and GP shape. Left: the folded pulse profiles from 1.2 GHz GB43 observations on MJD 55352 (top) and MJD 55532 (middle), and the difference between them (bottom). The profiles are scaled so that the area under the main pulse for both profiles is the same. As can be seen from the bottom plot, the difference is on the order of the rms noise, so there is no statistically significant change in pulse shape. Right: the average GP profiles from $1.2 \mathrm{GHz}$ GB43 observations on MJD 55352 (top) and MJD 55532 (middle), and the difference between them (bottom). This difference is not significant as there is a larger variation in average GP shape between pre-flare days. The profiles are also scaled so that the area under the main pulse for both profiles is the same.

Table 6

Maximum Correlation/Anticorrelation for Each Frequency at Each Energy Cut, and the Time Lags at which They Occur

\begin{tabular}{|c|c|c|c|c|c|c|}
\hline $\begin{array}{l}\text { Energy } \\
\text { Cut }\end{array}$ & $\begin{array}{l}\text { Frequency } \\
(\mathrm{MHz})\end{array}$ & $\mathrm{MP} / \mathrm{IP}$ & $\begin{array}{c}\text { Max } \\
\text { Correlation }(\sigma)\end{array}$ & $\begin{array}{c}\text { Time Lag } \\
\text { (Spin Periods) }\end{array}$ & $\begin{array}{c}\text { Max Anti- } \\
\text { correlation }(\sigma)\end{array}$ & $\begin{array}{c}\text { Time Lag } \\
\text { (Spin Periods) }\end{array}$ \\
\hline \multirow[t]{6}{*}{$100 \mathrm{MeV}$} & \multirow{2}{*}{ Pre-flare 1200} & MP & +1.4 & 30000 & -0.9 & 30 \\
\hline & & IP & +2.1 & 200000 & -1.7 & 300 \\
\hline & \multirow[t]{2}{*}{330} & MP & +0.2 & 10000 & -2.1 & 200 \\
\hline & & IP & +0.5 & 30 & -1.5 & 1000 \\
\hline & \multirow{2}{*}{ Post-flare 1200} & MP & +1.5 & 40 & -2.4 & 20000 \\
\hline & & IP & +1.9 & 500 & -2.3 & 30000 \\
\hline \multirow[t]{6}{*}{$\overline{500 \mathrm{MeV}}$} & \multirow[t]{2}{*}{ Pre-flare 1200} & MP & +2.3 & 1 & -0.4 & 8000 \\
\hline & & IP & +1.5 & 20000 & -1.5 & 700 \\
\hline & \multirow[t]{2}{*}{330} & MP & +0.1 & 6000 & -1.7 & 200 \\
\hline & & IP & -0.1 & 300 & -1.7 & 2000 \\
\hline & \multirow[t]{2}{*}{ Post-flare 1200} & MP & +0.8 & 200 & -3.5 & 20000 \\
\hline & & IP & +1.0 & 200 & -2.7 & 30000 \\
\hline \multirow[t]{6}{*}{$1 \mathrm{GeV}$} & \multirow[t]{2}{*}{ Pre-flare 1200} & MP & +3.2 & 1 & -0.1 & 60 \\
\hline & & IP & +2.0 & 5000 & -1.2 & 200 \\
\hline & \multirow[t]{2}{*}{330} & MP & +0.8 & 6000 & -0.9 & 10 \\
\hline & & IP & +0.9 & 6000 & -1.1 & 100 \\
\hline & \multirow{2}{*}{ Post-flare 1200} & MP & +1.9 & 60 & -0.4 & 1 \\
\hline & & IP & +2.1 & 3000 & -0.7 & 10 \\
\hline
\end{tabular}

Note. Note that the maximum correlation for the $330 \mathrm{MHz}$ IP with the $500 \mathrm{MeV}$ energy cut is negative.

during an observation as there were real $\gamma$-ray photons, we used this large time lag to make sure that there were the same number of real and randomized correlations once all of the $\gamma$-ray photons were included. The mean and standard deviation of the randomized correlations were calculated by randomly assigning $\gamma$-ray photon arrival times within GTIs and correlating them with GP TOAs. Figure 9 shows the results of this correlation analysis. The random correlations are the mean of 10,000 trials, and the error bars represent one standard deviation. The largest deviation from the mean is a $2.4 \sigma$ anticorrelation between $\gamma$-ray photons and post-flare 1.2 GHz MP GPs at a time lag of 20,000 spin periods, while the largest correlation is $2.1 \sigma$ for the 
pre-flare IP at $1.2 \mathrm{GHz}$ at a time lag of 200,000 spin periods. The maximum correlation/anticorrelation for each frequency at each energy cut is shown in Table 6. For both the MP and IP for each of the three data sets (pre-flare $1.2 \mathrm{GHz}, 330 \mathrm{MHz}$, post-flare $1.2 \mathrm{GHz}$ ), there are at most two time lags where the correlation exceeds $2 \sigma$. There are a total of 49 time lags included in the correlation, so two time lags are only four percent of the total data, while we statistically expect five percent of the data to have correlations beyond $2 \sigma$. Given the low significance and large time lag of these results, they do not provide any compelling case for a physical origin.

Selecting only $\gamma$-ray photons above $500 \mathrm{Mev}$ resulted in a total of 119 photons. The maximum correlation for this more restricted set was $2.3 \sigma$ at a time lag of one spin period for pre-flare MP at $1.2 \mathrm{GHz}$. The maximum anticorrelation was $3.5 \sigma$ at a time lag of 20,000 spin periods for the post-flare MP at $1.2 \mathrm{GHz}$. No more than one time lag for any data set had a correlation over $2 \sigma$, so those measurements above $2 \sigma$ are statistically insignificant. Statistically, only half of a percent of the data should be above $3 \sigma$, and two percent of our data have a $3.5 \sigma$ significance. However, this is likely due to the coarseness of our time lags. Given more time lags, we would expect this significance to drop below $3 \sigma$.

Only $65 \gamma$-ray photons had energies above $1 \mathrm{GeV}$. The maximum correlation occurred in the $1.2 \mathrm{GHz}$ pre-flare MP with a $3.2 \sigma$ significance at a time lag of one spin period. The maximum anticorrelation was $1.2 \sigma$, which occurred in the $1.2 \mathrm{GHz}$ pre-flare IP at a time lag of 200 spin periods. As with the $500 \mathrm{MeV}$ energy cut, we only see at most one time lag with above $2 \sigma$, which is insignificant. Similarly, the $3.2 \sigma$ significance is likely due to the coarseness of our time lags, and would likely drop given more time lags.

\section{CRAB NEBULA $\gamma$-RAY FLARE}

The recent $\gamma$-ray flare from the Crab Nebula was detected by the AGILE satellite above $100 \mathrm{MeV}$ (Tavani et al. 2011). Elevated $\gamma$-ray flux was observed from MJDs 55457-55461. No variations in pulse shape were found at $\gamma$-ray (Hays et al. 2010), X-ray (Tavani et al. 2011), or radio (Espinoza et al. 2010) energies. Espinoza et al. (2010) also found no increase in pulsed radio flux, glitches, or changes in DM around the date of the flare. Our closest observations before the flare were on MJD 55412 at $330 \mathrm{MHz}$ and MJD 55352 at $1.2 \mathrm{GHz}$, and our first observation after the flare was on MJD 55516 at $1.2 \mathrm{GHz}$.

We looked for changes in the average pulse profile, GP shape, power-law index, and $\gamma$-ray correlation in our pre- and post-flare 1.2 $\mathrm{GHz}$ data. We found no significant differences in the pulse profile shape and the average GP shape (Figure 10) before and on four days about two months after the flare. The pre-flare MP power-law indices are in the range $2.1 \pm 0.3$ to $3.1 \pm 0.2$, while the post-flare power-law indices for the MP and IP are $2.56 \pm$ 0.05 to $2.93 \pm 0.05$ and $2.4 \pm 0.4$ to $2.81 \pm 0.03$, respectively. We were unable to calculate power-law indices for the IP preflare due to low IP GP statistics. The largest anticorrelation between $\gamma$-ray photons and GPs was seen in the post-flare data, but it is still on the order of correlations/anticorrelations seen in pre-flare data, both at $1.2 \mathrm{GHz}$ and $330 \mathrm{MHz}$.

\section{CONCLUSIONS}

We compared GB43 and GBT GPs and found that 3\% of MP GPs and $5 \%$ of IP GPs at $1.2 \mathrm{GHz}$ were simultaneously detected at $8.9 \mathrm{GHz}$. The probability of either of these events occurring by chance is zero. Also, an additional four IP GPs at 1.2 GHz were within one $\mu$ s of an IP GP at $8.9 \mathrm{GHz}$, with a zero percent chance probability. This may suggest that, although the folded profiles are much different at the two frequencies, the emission mechanism is similar. However, the low percentage of low- and high-frequency IP GPs within one $\mu$ s could mean that the IP emission mechanism is different at higher frequencies, especially since the high-frequency IP is shifted by $10 \mathrm{deg}$.

Long observations allowed us to collect the largest sample of GPs to date, which we then used to calculate power-law indices for fits to amplitude distributions. These power-law indices agree with previously published values. A comparison of these indices to other source classes shows that GP emission is not exactly related to emission from other source classes and most closely matches the emission from magnetars and RRATS.

We found no significant correlations between GB43 GPs and Fermi $\gamma$-ray photons in the energy range $0.1-100 \mathrm{GeV}$. There are only a few correlations/anticorrelations in the MP and IP at both frequencies. They occur at different time lags and are within $2.5 \sigma$ of the mean for a correlation with randomized data. This suggests that although increased pair production in the magnetosphere may contribute to GP occurrence, it is not a dominant factor. More likely possibilities for GP generation are increased coherence or changes in beaming.

Multifrequency correlations have been searched for previously. Shearer et al. (2003) found a 3\% increase in the brightness of optical pulses at the time of GPs and Collins et al. (2012) found a slight correlation between GPs and enhanced optical pulses, which supports our $\sim 2 \sigma$ correlations/anticorrelations in suggesting that there are small fluctuations in the magnetospheric particle density during GPs. The anticorrelations, however, would suggest that increased particle density would lead to more radio emission and less $\gamma$-ray emission, which does not make sense in the context of Lyutikov's theory. Since the significance of the anticorrelations are on the order of the significance of the correlations, we assume that these are insignificant. Lundgren et al. (1995) found that the $\gamma$-ray flux does not vary by more than 2.5 times the average flux during a GP. Bilous et al. (2011) ruled out a strong correlation between GPs at $8.9 \mathrm{GHz}$ and $\gamma$-ray photons above $100 \mathrm{MeV}$. They were able to put an upper limit on the $\gamma$-ray flux during IP GPs of 8-16 times the average pulsed flux, suggesting that there still might be a slight correlation between GPs and $\gamma$-ray photons. We could not carry out the same experiment because we did not have sufficient $\gamma$-ray photons to compare the $\gamma$-ray profile made with $\gamma$-ray photons around GPs with the $\gamma$-ray profile excluding $\gamma$-ray photons around GPs.

The recent $\gamma$-ray flare from the Crab Nebula occurred during the span of our observations, so we were able to compare the behavior of the Crab pulsar before and after the flare. We found no significant changes in pulse shape, power-law index, or $\gamma$-ray correlation, suggesting that there was no change in the properties of the pulsar during the flare.

The National Radio Astronomy Observatory is a facility of the National Science Foundation operated under cooperative agreement by Associated Universities, Inc. This work was supported by NASA Fermi Grant NNX10AD14G and a WV EPSCoR grant.

\section{REFERENCES}

Abdo, A. A., Ackermann, M., Ajello, M., et al. 2010, ApJ, 708, 1254 Allen, C. W. 1973, Astrophysical Quantities (3rd ed.; London: Athlone) Argyle, E., \& Gower, J. F. R. 1972, ApJ, 175, L89 
Atwood, W. B., Abdo, A. A., Ackermann, M., et al. 2009, ApJ, 697, 1071 Bednarek, W., \& Idec, W. 2011, MNRAS, 414, 2229

Bhat, N. D. R., Tingay, S. J., \& Knight, H. S. 2008, ApJ, 676, 1200

Bhat, N. D. R., Wayth, R. B., Knight, H. S., et al. 2007, ApJ, 665, 618

Bietenholz, M. F., Kassim, N., Frail, D. A., et al. 1997, ApJ, 490, 291

Bilous, A. V., Kondratiev, V. I., McLaughlin, M. A., et al. 2011, ApJ, 728, 110

Bykov, A. M., Pavlov, G. G., Artemyev, A. V., \& Uvarov, Y. A. 2012, MNRAS, 421, L67

Cerutti, B., Uzdensky, D. A., \& Begelman, M. C. 2012, ApJ, 746, 148

Collins, S., Shearer, A., Stappers, B., et al. 2012, in IAU Symp 285, New Horizons in Time-Domain Astronomy, ed. E. R. Griffin, R. J. Hanisch, \& R. L. Seaman (Cambridge: Cambridge Univ. Press), 296

Cordes, J. M., Bhat, N. D. R., Hankins, T. H., McLaughlin, M. A., \& Kern, J. 2004, ApJ, 612, 375

Cordes, J. M., \& McLaughlin, M. A. 2003, ApJ, 596, 1142

DuPlain, R., Ransom, S., Demorest, P., et al. 2008, in Proc. SPIE 7019, Advanced Software and Control for Astronomy II, ed. A. Bridger \& N. M. Radziwill (Bellingham, WA: SPIE)

Espinoza, C. M., Jordan, C., Stappers, B. W., et al. 2010, ATel, 2889, 1

Hankins, T. H., \& Eilek, J. A. 2007, ApJ, 670, 693

Hays, E., Buehler, R., D’Ammando, F., Grove, J. E., \& Ray, P. S. 2010, ATel, 2879, 1

Hotan, A. W., van Straten, W., \& Manchester, R. N. 2004, PASA, 21, 302

Istomin, Y. N. 2004, in IAU Symp. 218, Young Neutron Stars and Their Environments, ed. F. Camilo \& B. M. Gaensler (San Francisco, CA: ASP), 369

Jessner, A., Popov, M. V., Kondratiev, V. I., et al. 2010, A\&A, 524, A60

Karuppusamy, R., Stappers, B. W., \& Lee, K. J. 2012, A\&A, 538, A7

Karuppusamy, R., Stappers, B. W., \& van Straten, W. 2010, A\&A, 515, A36

Kramer, M., Johnston, S., \& van Straten, W. 2002, MNRAS, 334, 523

Kuz'min, A. D., Belyatsky, Y. A., Dumsky, D. V., et al. 2011, Astron. Rep., 55,416

Langston, G. 2007, Highlights Astron., 14, 367
Lorimer, D. R., Yates, J. A., Lyne, A. G., \& Gould, D. M. 1995, MNRAS, 273,411

Lundgren, S. C. 1994, PhD thesis, Cornell Univ.

Lundgren, S. C., Cordes, J. M., Ulmer, M., et al. 1995, ApJ, 453, 433

Lyne, A. G., Pritchard, R. S., \& Graham-Smith, F. 1993, MNRAS, 265, 1003

Lyutikov, M. 2007, MNRAS, 381, 1190

Lyutikov, M., Blandford, R. D., \& Machabeli, G. 1999, MNRAS, 305, 338

Machabeli, G. Z., \& Usov, V. V. 1979, Sov. Astron. Lett., 5, 238

Majid, W. A., Naudet, C. J., Lowe, S. T., \& Kuiper, T. B. H. 2011, ApJ, 741,53

Moffett, D. A. 1997, PhD thesis, New Mexico Inst. Mining Technology

Moffett, D. A., \& Hankins, T. H. 1996, ApJ, 468, 779

Parsons, A., Werthimer, D., Backer, D., et al. 2009, The Astronomy and Astrophysics Decadal Survey 2010, 21

Petrova, S. A. 2006, Chin. J. Astron. Astrophys. Suppl., 6, 020000

Petrova, S. A. 2009, MNRAS, 395, 1723

Popov, M. V., Soglasnov, V. A., Kondratiev, V. I., et al. 2006, Astron. Rep., 50,55

Popov, M., Soglasnov, V., Kondratiev, V., et al. 2009, PASJ, 61, 1197

Popov, M. V., \& Stappers, B. 2007, A\&A, 470, 1003

Popov, M. V., Soglasnov, V. I., Kondratiev, A. V., et al. 2008, Astron. Rep., 52, 900

Rankin, J. M., Comella, J. M., Craft, H. D., Jr., et al. 1970, ApJ, 162, 707

Rickett, B. J., \& Lyne, A. G. 1990, MNRAS, 244, 68

Ritchings, R. T. 1976, MNRAS, 176, 249

Scargle, J. D. 1982, ApJ, 263, 835

Serylak, M., Stappers, B. W., Weltevrede, P., et al. 2009, MNRAS, 394, 295

Shearer, A., Stappers, B., O'Conner, P., et al. 2003, Science, 301, 493

Smirnova, T. V., \& Logvinenko, S. V. 2009, Astron. Rep., 53, 334

Staelin, D. H., \& Reifenstein, E. C., III. 1968, Science, 162, 1481

Tavani, M., Bulgarelli, A., Vittorini, V., et al. 2011, Science, 331, 736

Weatherall, J. C. 1998, ApJ, 506, 341

Zhuravlev, V. I., Popov, M. V., Kondratiev, V. I., et al. 2011, Astron. Rep., 55,724 